\title{
POSITIVE SPECIALIZATIONS OF SYMMETRIC GROTHENDIECK POLYNOMIALS
}

\author{
DAMIR YELIUSSIZOV
}

\begin{abstract}
It is a classical fundamental result that Schur-positive specializations of the ring of symmetric functions are characterized via totally positive functions whose parametrization describes the Edrei-Thoma theorem. In this paper, we study positive specializations of symmetric Grothendieck polynomials, $K$-theoretic deformations of Schur polynomials.
\end{abstract}

\section{INTRODUCTION}

Characterization of Schur-positive specializations of the ring of symmetric functions relies on a fundamental result in the theory of total positivity, the famous Edrei-Thoma theorem [Edr52, Tho64]. It became foundational to representation theory of the infinite symmetric group [Tho64, VK81, Ker03, BO17]. In combinatorics, positive specializations of Schur functions provide a powerful tool in enumeration of plane partitions [Sta99]. In probability, besides connections with the Plancherel measure on partitions [VK85], they are important in Schur processes [OR03].

In Schubert calculus, Schur polynomials relate to the cohomology of Grassmannians (e.g. [Ful97]). Similarly, $K$-theoretic Schubert calculus gives rise to certain deformations of Schur polynomials. These objects are known as Grothendieck polynomials [LS83, FK94]. They bear many similarities with the Schur basis but have significant differences. For instance, they are inhomogeneous as oppose to Schur functions. In combinatorial $K$-theory, underlying structures behind such deformations usually become more complicated, see e.g. [Buc02, Buc05, LP07, PP18, TY09, Yel17, Yel19].

1.1. Overview of main results. In this paper, we study positivity of $K$-theoretic deformations of Schur polynomials. Namely, we study positive specializations for three classes of symmetric Grothendieck polynomials (see definitions later):

(F1) the 'positive' basis $\left\{\tilde{G}_{\lambda}\right\}$

(F2) the usual basis $\left\{G_{\lambda}\right\}$ with alternating signs

(F3) the dual basis $\left\{g_{\lambda}\right\}$

A main feature of the family (F1) is that its structure constants and monomial expansions are all positive. It turns out that characterization of positive specializations for $\left\{\tilde{G}_{\lambda}\right\}$ is more difficult than for (F2). We are able to solve this problem for the families (F1), (F2) via transition to Schurpositive specializations. This might seem surprising, especially for (F1), as Schur functions expand non-positively in $\left\{\tilde{G}_{\lambda}\right\}$. We also show that positive specializations for (F1) characterize boundary of a certain filtered Young graph. As for the polynomials (F3), we describe a class of positive specializations and conjecture that it is complete. We also discuss how these specializations produce two analogues of the Plancherel measure on partitions: one is naturally related to the corner growth model in probability (e.g. [Joh00, Rom15]), another is the Plancherel-Hecke measure [TY11]. 
Let us now summarize the background and main results in more detail.

1.2. The Edrei-Thoma theorem. A fundamental result in the theory of total positivity is the theorem proved by Edrei [Edr52] and Thoma [Tho64], which characterizes totally nonnegative Toeplitz matrices. It was originally conjectured by Schoenberg [Sch34].

A matrix is called totally nonnegative if all its minors are nonnegative reals. A formal power series $A(z)=1+\sum_{n=1}^{\infty} a_{n} z^{n} \in \mathbb{R}[[z]]$ is a totally positive function if the associated (infinite, upper triangular) Toeplitz matrix $\left[a_{i-j}\right]_{i, j \geq 0}$ is totally nonnegative (where $a_{0}=1$ and $a_{n}=0$ for $n<0)$. The sequence $\left\{a_{n}\right\}$ is then called Pólya frequency sequence and every polynomial $A_{N}(z)=1+\sum_{n=1}^{N} a_{n} z^{n}$ (for $N=1,2, \ldots$ ) has only negative real roots, which is the AissenSchoenberg-Whitney theorem [ASW52].

Theorem 1.1 (Edrei-Thoma). $A(z)=1+\sum_{n=1}^{\infty} a_{n} z^{n}$ is a totally positive function if and only if

$$
A(z)=e^{\gamma z} \prod_{n=1}^{\infty} \frac{1+\beta_{n} z}{1-\alpha_{n} z}
$$

for nonnegative real parameters $\left\{\alpha_{n}\right\},\left\{\beta_{n}\right\}$, and $\gamma$ such that $\sum_{n}\left(\alpha_{n}+\beta_{n}\right)<\infty$.

Let $\Lambda$ be the ring of symmetric functions. One of the most important bases of $\Lambda$ is given by Schur functions $\left\{s_{\lambda}\right\}$. A homomorphism (specialization) $\rho: \Lambda \rightarrow \mathbb{R}$ is called Schur-positive if $\rho\left(s_{\lambda}\right) \geq 0$ for all partitions $\lambda$. The ring $\Lambda$ is a polynomial ring with generators $\left\{h_{n}\right\}$ of complete homogeneous symmetric functions. Thus any homomorphism of $\Lambda$ can be specified via values of $h_{n}$. Using the Jacobi-Trudi identity $s_{\lambda}=\operatorname{det}\left[h_{\lambda_{i}-i+j}\right]$, the Edrei-Thoma theorem equivalently characterizes Schur-positive specializations as follows.

Theorem 1.2. A homomorphism $\rho: \Lambda \rightarrow \mathbb{R}$ is Schur-positive if and only if

$$
\rho(H(z)):=1+\sum_{n=1}^{\infty} \rho\left(h_{n}\right) z^{n}=e^{\gamma z} \prod_{n=1}^{\infty} \frac{1+\beta_{n} z}{1-\alpha_{n} z}
$$

for nonnegative reals $\left\{\alpha_{n}\right\},\left\{\beta_{n}\right\}$, and $\gamma$ such that $\sum_{n}\left(\alpha_{n}+\beta_{n}\right)<\infty$. Equivalently, $\rho(H(z))$ is a totally positive function, where $H(z)$ is defined in (2).

An important specialization of $\Lambda$ is the Plancherel specialization $\pi$ which corresponds to the parameters $\gamma=1, \alpha_{k}=\beta_{k}=0$, and for which $\pi\left(s_{\lambda}\right)=f^{\lambda} / n$ !, where $f^{\lambda}$ is the number of standard Young tableaux (SYT) of shape $\lambda \vdash n$, or the dimension of an irreducible representation (indexed by $\lambda$ ) of the symmetric group $S_{n}$.

A relationship between totally positive functions and characters of the infinite symmetric group $S_{\infty}$ was made by Thoma [Tho64]. Vershik and Kerov [VK81] interpreted the parameters $\alpha_{n}, \beta_{n}$ asymptotically as normalized row and column lengths in growing partitions, describing characters of $S_{\infty}$. Original proofs of Edrei and Thoma used deep results from complex analysis, whereas Vershik and Kerov's approach relied on asymptotic representation theory. Note also that the difficult part of Theorem 1.2(or 1.1) is the only if direction, i.e. if a homomorphism is positive then it satisfies a given parametrization.

Similar positivity results are known for other classes of symmetric functions and generalizations of Schur polynomials, such as Jack symmetric functions [KOO98], Schur- $P, Q$ functions [Naz90], some general settings in [BO00], and a recent solution of Kerov's conjecture on positive specializations 
of Macdonald polynomials [Mat19]. We refer to [BO17] for more on the Edrei-Thoma theorem and representation theory of the infinite symmetric group, including background and many references therein.

1.3. Symmetric Grothendieck polynomials. Symmetric (or stable) Grothendieck polynomials are considered as a $K$-theoretic deformation of Schur polynomials. They were first studied by Fomin and Kirillov [FK94]. We begin by defining positive symmetric Grothendieck polynomials $\left\{\tilde{G}_{\lambda}\right\}$ by the following combinatorial formula due to Buch [Buc02]:

$$
\tilde{G}_{\lambda}=\tilde{G}_{\lambda}\left(x_{1}, x_{2}, \ldots\right):=\sum_{T \in S V T(\lambda)} \prod_{i \geq 1} x_{i}^{\# i \text { in } T},
$$

where the sum runs over shape $\lambda$ set-valued tableaux (SVT), a generalization of semistandard Young tableaux (SSYT) so that boxes may contain sets of integers (for precise definitions see Sec. 3). One can see that $\tilde{G}_{\lambda}=s_{\lambda}+\{$ higher degree terms $\} \in \hat{\Lambda}$, where $\hat{\Lambda}$ is the completion of $\Lambda$, that includes infinite linear combinations of basis elements. For example,

$$
\tilde{G}_{(1)}=e_{1}+e_{2}+e_{3}+\ldots \quad \text { or } \quad 1+\tilde{G}_{(1)}=\prod_{n=1}^{\infty}\left(1+x_{n}\right)
$$

where $e_{k}$ is the $k$ th elementary symmetric function.

Crucially, for all partitions $\mu, \nu$, the product

$$
\tilde{G}_{\mu} \cdot \tilde{G}_{\nu}=\sum_{\lambda} c_{\mu \nu}^{\lambda} \tilde{G}_{\lambda}, \quad|\lambda| \geq|\mu|+|\nu|, \quad c_{\mu \nu}^{\lambda} \in \mathbb{Z}_{\geq 0}
$$

expands as a finite sum. The nonnegative integers $c_{\mu \nu}^{\lambda}$ are generalized Littlewood-Richardson (LR) coefficients as in the lowest degree case $|\lambda|=|\mu|+|\nu|$ they become just the usual LR coefficients corresponding to product of Schur functions. This was proved by Buch [Buc02] via an explicit combinatorial LR rule for $c_{\mu \nu}^{\lambda}$. This finite expansion property can also be seen without any combinatorial interpretation of $c_{\mu \nu}^{\lambda}$ but conceptually on symmetric functions level [Yel19].

In light of the multiplication rule (1) one defines the commutative ring

$$
\Gamma:=\bigoplus_{\lambda} \mathbb{R} \cdot \tilde{G}_{\lambda}
$$

with a formal basis $\left\{\tilde{G}_{\lambda}\right\}$. The ring $\Gamma$ is related to $K$-theory of Grassmannians and we refer to [Buc02] for background. As is also mentioned there, the ring $\Gamma$ has somewhat unclear structure. For instance, it is not isomorphic to $\Lambda$ (but as completions $\hat{\Lambda} \cong \hat{\Gamma}$ ); we also do not know if it is a polynomial ring. Buch conjectured that any $\tilde{G}_{\lambda}$ is a polynomial in the elements $\tilde{G}_{R}$ for rectangular partitions $R \subset \lambda$; this would imply that the localization ring generated by $\Gamma$ and $q=\left(1+\tilde{G}_{(1)}\right)^{-1}$ is generated by the elements $\left\{\tilde{G}_{(n)}\right\},\left\{\tilde{G}_{\left(1^{n}\right)}\right\}$, and $q$ [Buc02].

1.4. Grothendieck-positive specializations. A homomorphism $\varphi: \Gamma \rightarrow \mathbb{R}$ is called G-positive (or Grothendieck-positive) if

$$
\varphi\left(\tilde{G}_{\lambda}\right) \geq 0 \text { for all partitions } \lambda .
$$

Say that $\varphi$ is normalized if $\varphi\left(\tilde{G}_{(1)}\right)=1$. We shall usually use the notation $\tilde{G}_{\lambda}(\varphi)$ for $\varphi\left(\tilde{G}_{\lambda}\right)$.

The main problem that we address and solve in this paper is the following. 
Problem 1.3. Describe $G$-positive specializations of the ring $\Gamma$.

One of the key results that we prove is the following transition theorem from Grothendieck to Schur positive specializations.

Theorem 1.4. Let $\varphi: \Gamma \rightarrow \mathbb{R}$ be a G-positive homomorphism of $\Gamma$. Then the map $\rho$ given by

$$
\rho: h_{n} \longmapsto \frac{\tilde{G}_{(n)}(\varphi)+\tilde{G}_{(n+1)}(\varphi)}{1+\tilde{G}_{(1)}(\varphi)} \quad n=1,2, \ldots
$$

defines a Schur-positive specialization of $\Lambda$.

Alternatively, the theorem states that we have the generating function for the elements $\left\{\tilde{G}_{(n)}(\varphi)\right\}$ :

$$
1+(z+1) \sum_{n=1}^{\infty} \tilde{G}_{(n)}(\varphi) z^{n-1}=(1+\delta) e^{\gamma z} \prod_{n=1}^{\infty} \frac{1+\beta_{n} z}{1-\alpha_{n} z}
$$

for nonnegative reals $\left\{\alpha_{n}\right\},\left\{\beta_{n}\right\}, \gamma$, and $\delta$ such that $\sum_{n}\left(\alpha_{n}+\beta_{n}\right)<\infty$. Here $\tilde{G}_{(1)}(\varphi)=\delta$ and for $z=-1$ (given it converges) we additionally get

$$
\delta=-1+e^{\gamma} \prod_{n=1}^{\infty} \frac{1+\alpha_{n}}{1-\beta_{n}}
$$

To prove this theorem we define certain auxiliary functions via Jacobi-Trudi-type formula (Sec. 4) and show that they are Grothendieck-positive, i.e. expand positively in the Grothendieck basis.

More precisely, we characterize normalized $G$-positive specializations as follows.

Theorem 1.5. Let $\varphi: \Gamma \rightarrow \mathbb{R}$ be a normalized $G$-positive homomorphism. Then the map

$$
\rho: h_{n} \longmapsto \frac{1}{2}\left(\tilde{G}_{(n)}(\varphi)+\tilde{G}_{(n+1)}(\varphi)\right) \quad n=1,2, \ldots
$$

defines a Schur-positive specialization of $\Lambda$ parametrized by nonnegative reals $\left\{\alpha_{n}\right\},\left\{\beta_{n}<1\right\}, \gamma$ such that

$$
\gamma=\log 2-\sum_{n} \log \left(1+\alpha_{n}\right)+\sum_{n} \log \left(1-\beta_{n}\right)
$$

Conversely, given a Schur-positive specialization parametrized by $\left\{\alpha_{n}\right\},\left\{\beta_{n}\right\}, \gamma$ as above, it extends to a normalized $G$-positive homomorphism of $\Gamma$.

1.5. Harmonic functions and boundary of a filtered Young's graph. Consider the (infinite) filtered Young graph $\widetilde{\mathbb{Y}}$ defined as follows:

(i) its vertices are labeled by partitions $\lambda$;

(ii) there is an arc $\lambda \rightarrow \mu$ iff $\mu / \lambda$ is a rook strip (i.e. no two boxes lie in the same row or column) As we will see, this graph encodes Pieri rules for Grothendieck polynomials.

Let $\mathcal{P}$ be the set of partitions. A function $\varphi: \mathcal{P} \rightarrow \mathbb{R}_{\geq 0}$ is called harmonic ${ }^{1}$ on the graph $\widetilde{\mathbb{Y}}$ if

$$
\varphi(\varnothing)=1 \quad \text { and } \quad \varphi(\lambda)=\sum_{\mu: \lambda \rightarrow \mu} \varphi(\mu)
$$

\footnotetext{
${ }^{1}$ Harmonic functions in Vershik-Kerov sense [VK81], usually defined for graded graphs [Ker03, BO00].
} 
Let $H(\widetilde{\mathbb{Y}})$ be the convex set of harmonic functions on $\widetilde{\mathbb{Y}}$ and let $\partial \widetilde{\mathbb{Y}} \subset H(\widetilde{\mathbb{Y}})$ be the set of extreme points of $H(\widetilde{\mathbb{Y}})$, or the boundary of $\widetilde{\mathbb{Y}}$ (i.e. the set of harmonic functions that are not expressible as nontrivial convex combinations of other harmonic functions).

For every function $\varphi \in H(\widetilde{\mathbb{Y}})$ define the linear functional $\hat{\varphi}: \Gamma \rightarrow \mathbb{R}$ such that $\hat{\varphi}\left(\tilde{G}_{\lambda}\right):=\varphi(\lambda)$. Then $G$-positive homomorphisms characterize the corresponding boundary. Namely, the set of linear functionals of the boundary $\partial \widetilde{\mathbb{Y}}$ coincides with the set of normalized $G$-positive homomorphisms. We have the following version of Vershik-Kerov "ring theorem" [KV80, BO17] for $\widetilde{\mathbb{Y}}$.

Theorem 1.6. We have: $\varphi \in \partial \widetilde{\mathbb{Y}}$ i.e. $\varphi$ is extreme if and only if $\hat{\varphi}$ is a normalized G-positive homomorphism of $\Gamma$.

The graph $\widetilde{\mathbb{Y}}$ is viewed as a filtered deformation of graded Young's lattice $\mathbb{Y}$ as its vertices $\lambda \in \mathcal{P}$ form a graded set ranked by $|\lambda|$ and arcs join vertices from lower to higher ranks. Note also that this graph is part of the Möbius deformation of Young's graph [PP18] (cf. [Yel19]), which is one of major examples of dual filtered graphs studied by Patrias and Pylyavskyy [PP18] as $K$-theoretic analogues of Fomin's dual graded graphs [Fom94] and Stanley's differential posets [Sta88].

1.6. Grothendieck polynomials with alternating signs. The functions $\left\{G_{\lambda}\right\}$ are usually defined with alternating signs in monomials [Len00, Buc02, LP07], namely as the functions

$$
G_{\lambda}\left(x_{1}, x_{2}, \ldots\right):=(-1)^{|\lambda|} \tilde{G}_{\lambda}\left(-x_{1},-x_{2}, \ldots\right) .
$$

We also define $\bar{G}$-positive homomorphisms $\varphi$ of $\Gamma$ satisfying $\varphi\left(G_{\lambda}\right) \geq 0$. In Sec. 6 we study and characterize such $\bar{G}$-positive specializations similarly as for $G$-positivity.

1.7. Dual Grothendieck polynomials. There is a basis $\left\{g_{\lambda}\right\}$ of $\Lambda$ that is dual to $\left\{G_{\lambda}\right\}$ via the Hall inner product for which Schur functions form an orthonormal basis. It was explicitly described via plane partitions by Lam and Pylyavskyy in [LP07]. In Sec. 7 we also define and describe a class of $g$-positive specializations of the ring $\Lambda$ (Proposition 7.5). We conjecture that this class is in fact complete, i.e. it describes all $g$-positive specializations.

\section{SCHUR-POSITIVE SPECIALIZATIONS}

2.1. Partitions and Young diagrams. A partition is a sequence $\lambda=\left(\lambda_{1} \geq \ldots \geq \lambda_{\ell}>0\right)$, where $\ell=\ell(\lambda)$ is the length of $\lambda$. Any partition can be represented as a Young diagram with $\lambda_{i}$ boxes in row $i$; equivalently, as the set $\left\{(i, j): 1 \leq i \leq \ell, 1 \leq j \leq \lambda_{i}\right\}$. The partition $\lambda^{\prime}$ is the conjugate of $\lambda$ obtained by transposing its diagram. We use English notation for drawing Young diagrams, index columns from left to right and rows from top to bottom. Let $\mathcal{P}$ be the set of partitions.

2.2. Specializations of the ring of symmetric functions. The ring $\Lambda$ of symmetric functions in the variables $\mathbf{x}=\left(x_{1}, x_{2}, \ldots\right)$ can be viewed as

$$
\Lambda \cong \mathbb{R}\left[h_{1}, h_{2}, \ldots\right] \cong \mathbb{R}\left[e_{1}, e_{2}, \ldots\right] \cong \mathbb{R}\left[p_{1}, p_{2}, \ldots\right]
$$

i.e. a polynomial ring with one of the following sets of generators:

$$
h_{n}:=\sum_{1 \leq i_{1} \leq \ldots \leq i_{n}} x_{i_{1}} \cdots x_{i_{n}}, \quad e_{n}:=\sum_{1 \leq i_{1}<\ldots<i_{n}} x_{i_{1}} \cdots x_{i_{n}}, \quad p_{n}:=\sum_{1 \leq i} x_{i}^{n}
$$

of complete homogeneous, elementary, or power sum symmetric functions. 
A specialization is any homomorphism $\Lambda \rightarrow \mathbb{R}$ and it can be defined by specifying generators. For any specializations $\rho_{1}, \rho_{2}: \Lambda \rightarrow \mathbb{R}$ we can define their union

$$
\rho=\left(\rho_{1}, \rho_{2}\right)=\rho_{1} \cup \rho_{2}
$$

via the power sum $\left\{p_{n}\right\}$ generators as follows:

$$
\rho\left(p_{n}\right):=\rho_{1}\left(p_{n}\right)+\rho_{2}\left(p_{n}\right) \text { for all } n=1,2, \ldots
$$

Note that we have

$$
H(z):=1+\sum_{n=1}^{\infty} h_{n} z^{n}=\prod_{n=1}^{\infty} \frac{1}{1-z x_{n}}=\exp \left(\sum_{n=1}^{\infty} \frac{p_{n}}{n} z^{n}\right)
$$

and hence

$$
\rho(H(z))=\rho_{1}(H(z)) \cdot \rho_{2}(H(z)) .
$$

From these generating function identities we have the following.

Lemma 2.1. Let $\rho, \rho_{1}, \rho_{2}: \Lambda \rightarrow \mathbb{R}$ be specializations of $\Lambda$. We have: $\rho=\left(\rho_{1}, \rho_{2}\right)$ if and only if $\rho(H(z))=\rho_{1}(H(z)) \cdot \rho_{2}(H(z))$.

2.3. Schur-positive specializations. The ring $\Lambda$ has a linear basis $\left\{s_{\lambda}\right\}$ of Schur functions that can be defined as follows

$$
s_{\lambda}\left(x_{1}, x_{2}, \ldots\right):=\sum_{T \in S S Y T(\lambda)} x^{T}
$$

where $S S Y T(\lambda)$ is the set of semistandard Young tableaux (SSYT) of shape $\lambda$, i.e. filings of the boxes of the Young diagram of $\lambda$ with positive integers weakly increasing in rows (from left to right) and strictly increasing in columns (from top to bottom); and $x^{T}=\prod_{i \geq 1} x_{i}^{a_{i}}$, where $a_{i}$ is the number of $i$ 's in tableau $T \in S S Y T(\lambda)$.

There is a standard involutive automorphism $\omega: \Lambda \rightarrow \Lambda$ given on generators by $\omega: h_{n} \mapsto e_{n}$ for all $n \geq 1$ and for which $\omega\left(s_{\lambda}\right)=s_{\lambda^{\prime}}$.

Definition 2.2. A homomorphism $\rho: \Lambda \rightarrow \mathbb{R}$ is called Schur-positive if $\rho\left(s_{\lambda}\right) \geq 0$ for all $\lambda$.

Definition 2.3. Let $\alpha, \beta, \gamma \geq 0$ be scalars. Define the following Schur-positive generators $\phi, \varepsilon, \pi$ given by:

- The specialization $\phi_{\alpha}$ :

$$
\phi_{\alpha}(H(z))=(1-\alpha z)^{-1}
$$

for which $\phi_{\alpha}\left(s_{\lambda}\right)=\alpha^{|\lambda|}$ if $\ell(\lambda)=1$ and 0 otherwise. Equivalently, it is just single variable substitution $x_{1} \mapsto \alpha, x_{k} \mapsto 0$ for $k \geq 2$.

- The specialization $\varepsilon_{\beta}$ :

$$
\varepsilon_{\beta}(H(z))=1+\beta z
$$

for which $\varepsilon_{\beta}\left(s_{\lambda}\right)=\beta^{|\lambda|}$ if $\lambda_{1}=1$ and 0 otherwise. Note that $\varepsilon_{\beta}=\phi_{\beta} \circ \omega$, i.e. a composition of $\phi$ with the involution $\omega$.

- The Plancherel specialization

$$
\pi_{\gamma}(H(z))=e^{\gamma z}
$$

for which $\pi_{\gamma}\left(s_{\lambda}\right)=\gamma^{n} f^{\lambda} / n$ ! for $\lambda \vdash n$, where $f^{\lambda}$ is the number of SYT of shape $\lambda$. Equivalently, it is given by $p_{1} \mapsto \gamma$ and $p_{k} \mapsto 0$ for $k \geq 2$. 
Note that union of Schur-positive specializations is also Schur-positive. This can be seen from the branching formulas

$$
\rho\left(s_{\lambda}\right)=\sum_{\mu} \rho_{1}\left(s_{\lambda / \mu}\right) \rho_{2}\left(s_{\mu}\right)=\sum_{\mu, \nu} c_{\mu \nu}^{\lambda} \rho_{1}\left(s_{\nu}\right) \rho_{2}\left(s_{\mu}\right) \geq 0
$$

if $\rho=\left(\rho_{1}, \rho_{2}\right)=\rho_{1} \cup \rho_{2}$ is a union of Schur-positive specializations.

Combining these facts with an observation made in Lemma 2.1, we obtain the following equivalent formulation for characterization (Theorem 1.2) of Schur-positive specializations.

Theorem 2.4 (Edrei-Thoma factorization form). A specialization $\rho: \Lambda \rightarrow \mathbb{R}$ is Schur-positive if and only if

$$
\rho=\pi_{\gamma} \cup \phi_{\alpha_{1}} \cup \phi_{\alpha_{2}} \cup \cdots \cup \varepsilon_{\beta_{1}} \cup \varepsilon_{\beta_{2}} \cup \ldots
$$

for nonnegative reals $\gamma,\left\{\alpha_{n}\right\},\left\{\beta_{n}\right\}$ such that $\sum_{n}\left(\alpha_{n}+\beta_{n}\right)<\infty$.

Remark 2.5. In terms of the power sum generators, Schur-positive specializations $\rho$ are given by

$$
\rho\left(p_{k}\right)=\pi_{\gamma}\left(p_{k}\right)+\sum_{n} \phi_{\alpha_{n}}\left(p_{k}\right)+\sum_{n} \varepsilon_{\beta_{n}}\left(p_{k}\right) \text { for } k \geq 1,
$$

or more precisely we have

$$
\rho: p_{1} \longmapsto \gamma+\sum_{n}\left(\alpha_{n}+\beta_{n}\right) \quad \text { and } \quad \rho: p_{k} \longmapsto \sum_{n}\left(\left(\alpha_{n}\right)^{k}+(-1)^{k-1}\left(\beta_{n}\right)^{k}\right) \text { for } k \geq 2 .
$$

The condition $\sum_{n}\left(\alpha_{n}+\beta_{n}\right)<\infty$ is necessary and sufficient for all $p_{k}$ to converge.

Remark 2.6. Product of generating functions that specialize $H(z)$ corresponds to product of associated Toeplitz matrices so that $(1-\alpha z)^{-1},(1+\beta z)$, $e^{\gamma z}$ generate totally positive functions $\rho(H(z))$.

\section{Symmetric Grothendieck Polynomials}

Let us give some notation for Young diagrams. We use definitions introduced in [Yel19].

Denote by $I(\lambda)$ the set of inner corner boxes of $\lambda$ and $i(\lambda)=\# I(\lambda)$. For partitions $\lambda \supset \mu$ define the following extension of skew shapes:

$$
\lambda / / \mu:=\lambda / \mu \cup I(\mu) .
$$

E.g. $(5331) / /(432)$ consists of the skew shape $(5331) /(432)=\{\square\}$ and $I(432)=\{\square\}$, see the picture.

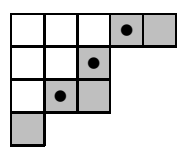

Denote by $a(\lambda / / \mu)$ the number of open boxes of $I(\mu)$ that do not lie in the same column with any box of $\lambda / \mu$. Equivalently, it is the number of columns of $\lambda / / \mu$ that are not columns of $\lambda / \mu$. For example, $a((5331 / /(432)))=2$, the boxes 0 in the picture.

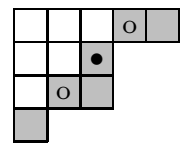




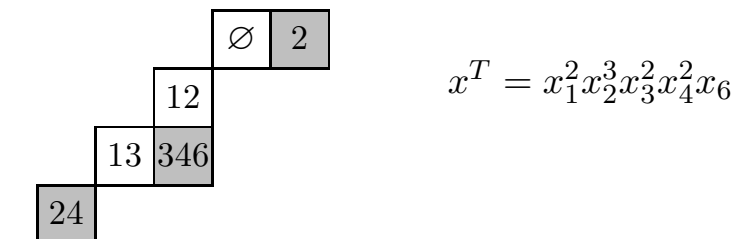

Figure 1. A set-valued tableau of shape $(5331) / /(432)=(5331) /(432) \cup I(432)$. The gray boxes is the skew shape $(5331) /(432)$; the white boxes is $I(432)$, corners of $(432)$. It is allowed to put $\varnothing$ in $I(\mu)$ but boxes of $\lambda / \mu$ must be nonempty.

Denote by $c(\lambda / \mu)$ and $r(\lambda / \mu)$ the number of (nonempty) columns and rows of $\lambda / \mu$, respectively. We say that $\lambda / \mu$ is a horizontal (resp. vertical) strip if $\lambda / \mu$ has no two boxes in the same column (resp. row). Say that $\lambda / \mu$ is a rook strip if no two boxes of $\lambda / \mu$ lie in the same row and column, equivalently when $|\lambda / \mu|=c(\lambda / \mu)=r(\lambda / \mu)$, e.g. (5331)/(432) is a rook strip, see Fig. 1 gray boxes.

Definition 3.1. A set-valued tableau (SVT) of shape $\lambda / / \mu=\lambda / \mu \cup I(\mu)$ is a filling of the boxes of $\lambda / / \mu$ by sets of positive integers such that

- numbering is semistandard, i.e. if we replace each set by any of its elements, then the numbers increase from left to right and from top to bottom;

- each box of $\lambda / \mu$ contains a non-empty set;

- each box of $I(\mu)$ contains a set (maybe empty).

Let $S V T(\lambda / / \mu)$ be the set of SVT of shape $\lambda / / \mu$. For $T \in S V T(\lambda / / \mu)$, define the corresponding monomial $x^{T}=\prod_{i \geq 1} x_{i}^{a_{i}}$, where $a_{i}$ is the number of $i$ 's in $T$. See the example in Fig. 1 .

We use the notation $\mathbf{x}=\left(x_{1}, x_{2}, \ldots\right)$ and $\mathbf{y}=\left(y_{1}, y_{2}, \ldots\right)$ for (infinite) sets of variables.

Definition 3.2. Symmetric Grothendieck polynomials $\tilde{G}_{\lambda / / \mu}$ are defined via the following series:

$$
\tilde{G}_{\lambda / / \mu}(\mathbf{x}):=\sum_{T \in S V T(\lambda / / \mu)} x^{T}
$$

In particular, $\tilde{G}_{\lambda / / \varnothing}=\tilde{G}_{\lambda}$ for straight shapes. It is easy to see that

$$
\tilde{G}_{(1)}=-1+\prod_{n}\left(1+x_{n}\right)=e_{1}+e_{2}+\ldots
$$

Note also that

$$
\tilde{G}_{\lambda / / \lambda}=\prod_{n}\left(1+x_{n}\right)^{i(\lambda)} \neq \tilde{G}_{\varnothing}=1 .
$$

Proposition 3.3 ([Yel19]). The following branching formula holds:

$$
\tilde{G}_{\lambda / / \mu}(\mathbf{x}, \mathbf{y})=\sum_{\nu} \tilde{G}_{\lambda / / \nu}(\mathbf{x}) \tilde{G}_{\nu / / \mu}(\mathbf{y})
$$

For a single variable $x$ we have

$$
\tilde{G}_{\lambda / / \mu}(x)= \begin{cases}(1+x)^{a(\lambda / / \mu)} x^{|\lambda / \mu|}, & \text { if } \lambda / \mu \text { is a horizontal strip } \\ 0, & \text { otherwise. }\end{cases}
$$


We also have the following Pieri rules first proved by Lenart [Len00]

$$
\tilde{G}_{(k)} \cdot \tilde{G}_{\lambda}=\sum_{\mu / \lambda \text { hor. strip }}\left(\begin{array}{c}
r(\mu / \lambda)-1 \\
|\mu / \lambda|-k
\end{array}\right) \tilde{G}_{\mu}
$$

and in particular the simple Pieri rule

$$
\tilde{G}_{(1)} \cdot \tilde{G}_{\lambda}=\sum_{\mu / \lambda \text { rook strip }} \tilde{G}_{\mu}
$$

We have $\tilde{G}_{\lambda}=s_{\lambda}+\{$ higher degree terms $\} \in \hat{\Lambda}$ are elements of the completion $\hat{\Lambda}$ of the ring $\Lambda$ consisting of unbounded degree elements. Since the lowest degree component is the Schur basis $\left\{s_{\lambda}\right\}$, the functions $\left\{\tilde{G}_{\lambda}\right\}$ are linearly independent. To describe transition coefficients with the Schur basis let us introduce two types of tableaux.

Definition 3.4 ([Len00, LP07]). Define the following tableaux:

- A strict elegant tableau of shape $\mu / \lambda$ is an SYT (i.e. strictly increasing in rows and columns) whose entries in row $i$ lie in $[1, i-1]$ for all $i$. Let $\mathrm{r}_{\mu / \lambda}$ be the number of strict elegant tableaux of shape $\mu / \lambda$. In particular, if $\mathrm{r}_{\mu / \lambda}>0$ then $\mu_{1}=\lambda_{1}$.

- An elegant tableau of shape $\mu / \lambda$ is an SSYT whose entries in row $i$ lie in $[1, i-1]$ for all $i$. Let $\mathrm{f}_{\mu / \lambda}$ be the number of elegant tableaux of shape $\mu / \lambda$. In particular, if $\mathrm{f}_{\mu / \lambda}>0$ then $\mu_{1}=\lambda_{1}$.

Then we have the following infinite expansions [Len00]

$$
\tilde{G}_{\lambda}=\sum_{\mu \supset \lambda} \mathrm{r}_{\mu / \lambda} s_{\mu}, \quad s_{\lambda}=\sum_{\mu \supset \lambda}(-1)^{|\mu / \lambda|} \mathrm{f}_{\mu / \lambda} \tilde{G}_{\mu} .
$$

As discussed in the introduction, for all $\mu, \nu$ the product

$$
\tilde{G}_{\mu} \cdot \tilde{G}_{\nu}=\sum_{\lambda} c_{\mu \nu}^{\lambda} \tilde{G}_{\lambda}, \quad|\lambda| \geq|\mu|+|\nu|, \quad c_{\mu \nu}^{\lambda} \in \mathbb{Z}_{+}
$$

expands as a finite sum; here $c_{\mu \nu}^{\lambda}$ are generalized LR coefficients [Buc02]. Hence we can define the commutative ring

$$
\Gamma:=\bigoplus_{\lambda} \mathbb{R} \cdot \tilde{G}_{\lambda}
$$

with a formal basis $\left\{\tilde{G}_{\lambda}\right\}$ and the product given by (7). There is an involutive automorphism $\tau: \Gamma \rightarrow \Gamma$ given by $\tau: \tilde{G}_{\lambda} \mapsto \tilde{G}_{\lambda^{\prime}}$ (see [Buc02, Yel17]). We have $\tau\left(\tilde{G}_{\lambda / / \mu}\right)=\tilde{G}_{\lambda^{\prime} / / \mu^{\prime}}$ (see [Yel19]).

We also have finite expansions (see [Buc02])

$$
\begin{gathered}
\tilde{G}_{\lambda}(\mathbf{x}, \mathbf{y})=\sum_{\mu, \nu} d_{\mu \nu}^{\lambda} \tilde{G}_{\mu}(\mathbf{x}) \tilde{G}_{\nu}(\mathbf{y}), \quad d_{\mu \nu}^{\lambda} \in \mathbb{Z}_{+} \\
\tilde{G}_{\lambda / / \mu}=\sum_{\nu} d_{\mu \nu}^{\lambda} \tilde{G}_{\nu} \in \Gamma .
\end{gathered}
$$

Note that the structure coefficients are the same in both expansions which follows from branching formulas for $\tilde{G}_{\lambda}$ (or Hopf-algebraic properties). 
3.1. Specializations of the ring $\Gamma$. A specialization of $\Gamma$ is any homomorphism $\Gamma \rightarrow \mathbb{R}$.

Definition 3.5. Let $\varphi_{1}, \varphi_{2}$ be homomorphisms of $\Gamma$. Define their union $\varphi=\left(\varphi_{1}, \varphi_{2}\right)=\varphi_{1} \cup \varphi_{2}$ such that for all $\lambda$ we have

$$
\tilde{G}_{\lambda}(\varphi)=\tilde{G}_{\lambda}\left(\varphi_{1}, \varphi_{2}\right)=\sum_{\nu} \tilde{G}_{\lambda / / \nu}\left(\varphi_{1}\right) \tilde{G}_{\nu}\left(\varphi_{2}\right)
$$

By the branching formula (3), it is well-defined and is compatible with union of specializations of $\Lambda$ as we discuss below.

Definition 3.6. Let $\rho$ be a specialization of $\Lambda$. Say that $\rho$ extends to a specialization of $\Gamma$ if $\tilde{G}_{\lambda}$ are well-defined as an image under $\rho$. Formally, if the homomorphism $\hat{\rho}: \Gamma \rightarrow \mathbb{R}$ given by

$$
\tilde{G}_{\lambda}(\hat{\rho}):=\sum_{\mu} \mathrm{r}_{\mu / \lambda} \rho\left(s_{\mu}\right)
$$

is well-defined. In particular, all such infinite sums converge.

Lemma 3.7. Let $\rho_{1}, \rho_{2}$ be specializations of $\Lambda$ that extend to specializations $\hat{\rho}_{1}, \hat{\rho}_{2}$ of $\Gamma$. Then the union $\rho=\left(\rho_{1}, \rho_{2}\right)$ of $\Lambda$ extends to the specialization $\hat{\rho}=\left(\hat{\rho}_{1}, \hat{\rho}_{2}\right)$ of $\Gamma$.

Proof. Follows from the branching formulas (3), (8) and Schur expansions (6). Namely, we have the following identities

$$
\tilde{G}_{\lambda}(\mathbf{x}, \mathbf{y})=\sum_{\mu, \nu} d_{\mu \nu}^{\lambda} \tilde{G}_{\mu}(\mathbf{x}) \tilde{G}_{\nu}(\mathbf{y})=\sum_{\mu, \nu} d_{\mu \nu}^{\lambda} \sum_{\eta} \mathrm{r}_{\eta / \mu} s_{\eta}(\mathbf{x}) \sum_{\kappa} \mathrm{r}_{\kappa / \nu} s_{\kappa}(\mathbf{y}) .
$$

On the other hand,

$$
\tilde{G}_{\lambda}(\mathbf{x}, \mathbf{y})=\sum_{\theta} \mathrm{r}_{\theta / \lambda} s_{\theta}(\mathbf{x}, \mathbf{y})=\sum_{\theta} \mathrm{r}_{\theta / \lambda} \sum_{\eta, \kappa} c_{\eta \kappa}^{\theta} s_{\eta}(\mathbf{x}) s_{\kappa}(\mathbf{y})
$$

Combining these identities by applying $\rho$ with the definition of unions we obtain

$$
\begin{aligned}
\tilde{G}_{\lambda}(\hat{\rho}) & =\sum_{\mu, \nu} d_{\mu \nu}^{\lambda} \tilde{G}_{\mu}\left(\hat{\rho}_{1}\right) \tilde{G}_{\nu}\left(\hat{\rho}_{2}\right) \\
(\operatorname{by}(11)) & =\sum_{\mu, \nu} d_{\mu \nu}^{\lambda} \sum_{\eta} \mathrm{r}_{\eta / \mu} \rho_{1}\left(s_{\eta}\right) \sum_{\kappa} \mathrm{r}_{\kappa / \nu} \rho_{2}\left(s_{\kappa}\right) \\
(\operatorname{by}(12)) & =\sum_{\theta} \mathrm{r}_{\theta / \lambda} \sum_{\eta, \kappa} c_{\eta \kappa}^{\theta} \rho_{1}\left(s_{\eta}\right) \rho_{2}\left(s_{\kappa}\right) \\
& =\sum_{\theta} \mathrm{r}_{\theta / \lambda} \rho\left(s_{\theta}\right)
\end{aligned}
$$

as needed.

\section{Grothendieck-Positive specializations}

Definition 4.1. A homomorphism $\varphi: \Gamma \rightarrow \mathbb{R}$ is called $G$-positive if $\tilde{G}_{\lambda}(\varphi) \geq 0$ for all $\lambda$.

Define the following positive cone in $\Gamma$ of nonnegative linear combinations of $\left\{\tilde{G}_{\lambda}\right\}$ :

$$
\Gamma_{+}:=\bigoplus_{\lambda} \mathbb{R}_{\geq 0} \cdot \tilde{G}_{\lambda} \subset \Gamma
$$


Note that it is closed under multiplication since products of symmetric Grothendieck polynomials expand positively in $\left\{\tilde{G}_{\lambda}\right\}$.

Lemma 4.2. Let $\varphi$ be a G-positive homomorphism of $\Gamma$. Then $\tilde{G}_{\lambda / / \mu}(\varphi) \geq 0$.

Proof. Follows from the fact $\tilde{G}_{\lambda / / \mu} \in \Gamma_{+}$is Grothendieck-positive, see (9).

Lemma 4.3. Let $\varphi_{1}, \varphi_{2}$ be G-positive homomorphisms of $\Gamma$. Then the union $\varphi=\left(\varphi_{1}, \varphi_{2}\right)$ is also G-positive.

Proof. Immediate from the definition 3.5 of union and previous lemma.

Consider the elements $H_{n} \in \Gamma_{+}$defined as follows

$$
H_{0}:=1+\tilde{G}_{(1)}, \quad H_{n}:=\tilde{G}_{(n)}+\tilde{G}_{(n+1)} \text { for } n>0, \text { and } H_{n}=0 \text { for } n<0 .
$$

For any partition $\mu$, define the function

$$
F_{\mu}:=\operatorname{det}\left[H_{\mu_{i}-i+j}\right]_{1 \leq i, j \leq \ell(\mu)} \in \Gamma
$$

To describe expansion of $F_{\mu}$ in the basis $\left\{\tilde{G}_{\lambda}\right\}$, we need to define the following types of tableaux.

Definition 4.4. A delegant tableau of shape $\lambda / \mu$ is a filling of the boxes of $\lambda / \mu$ with positive integers such that

- the entries weakly decrease in rows from left to right and strictly decrease from top to bottom

- the elements in row $i$ lie in $\left[\lambda_{i}, \ell+1+\mu_{i}-i\right]$, where $\ell=\ell(\mu)$.

Let $d_{\lambda / \mu}$ be the number of delegant tableaux of shape $\lambda / \mu$. Note that from the second property we must have $\lambda_{1} \leq \ell+\mu_{1}$ and $\ell(\lambda)=\ell=\ell(\mu)$. Note also that for any fixed $\mu$ there is only finitely many $\lambda$ such that $\mathrm{d}_{\lambda / \mu}>0$.

The core result that allows us to describe $G$-positive specializations is the following theorem showing that the function $F_{\mu}$ is Grothendieck-positive.

Theorem 4.5. We have the following expansion

$$
F_{\mu}=\sum_{\nu} \mathrm{d}_{\nu / \mu} \tilde{G}_{\nu}
$$

where $\mathrm{d}_{\nu / \mu}$ is the number of delegant tableaux of shape $\nu / \mu$. In particular, $F_{\mu} \in \Gamma_{+}$.

An application of this theorem is the following.

Theorem 4.6. Let $\varphi$ be a G-positive homomorphism of $\Gamma$. Then the Toeplitz matrix

$$
H(\varphi):=\left[H_{i-j}(\varphi)\right]_{i, j \geq 0}
$$

is totally nonnegative.

Proof. First note that the diagonal elements $H_{0}(\varphi)=1+\tilde{G}_{1}(\varphi)>0$ are positive and since the matrix $H(\varphi)$ is upper triangular, the principal minors $\left[H_{i-j}(\varphi)\right]_{0 \leq i, j \leq n}$ are positive for all $n \geq 0$. For all partitions $\lambda$, the minors $\operatorname{det}\left[H_{\lambda_{i}-i+j}(\varphi)\right]=F_{\lambda}(\varphi) \geq 0$ are nonnegative by Theorem 4.5. In particular, all minors occupying several consecutive columns are nonnegative. Nonnegativity of 
all these minors and positivity of the principal minors is sufficient for the matrix $H$ to be totally nonnegative (see the nonnegativity criterion [GP92, Thm. 3.2]; see also [FZ00] for total positivity tests).

To prove Theorem 4.5 we need some preparatory lemmas.

Lemma 4.7. We have $H_{n}=H_{0} \cdot h_{n}$ for all $n \geq 0$. Equivalently, we have the generating series

$$
\sum_{n=0}^{\infty} H_{n} z^{n}=1+(z+1) \sum_{n=1}^{\infty} \tilde{G}_{(n)} z^{n-1}=\prod_{n=1}^{\infty} \frac{1+x_{n}}{1-z x_{n}}
$$

Proof. It is known that (see [Len00])

$$
\tilde{G}_{(n)}=\sum_{i=0}^{\infty} s_{(n-1 \mid i)}
$$

where $s_{(a \mid b)}=s_{\left(a+1,1^{b}\right)}$ is the Schur function of hook shape. On the other hand, using the Pieri rule for Schur functions we get

$$
H_{0} \cdot h_{n}=\left(1+\tilde{G}_{(1)}\right) \cdot h_{n}=\sum_{i=0}^{\infty} e_{i} h_{n}=\sum_{i=0}^{\infty} s_{(n-1 \mid i)}+s_{(n \mid i)}=\tilde{G}_{(n)}+\tilde{G}_{(n+1)}=H_{n} .
$$

The generating function identity follows as well.

Lemma 4.8. The following determinantal formula holds

$$
\mathrm{d}_{\nu / \mu}=\operatorname{det}\left[\left(\begin{array}{c}
\ell-i+1 \\
\nu_{i}-i-\mu_{j}+j
\end{array}\right)\right]_{1 \leq i, j \leq \ell},
$$

where $\ell=\ell(\mu)=\ell(\nu)$.

Proof. A standard exercise on the Lindström-Gessel-Viennot Lemma, see e.g. [GV89, Thm. 15].

Lemma 4.9. The following determinantal formula holds

$$
\mathrm{f}_{\nu / \lambda}=\operatorname{det}\left[\left(\begin{array}{c}
\nu_{i}-\lambda_{j}+j-2 \\
\nu_{i}-i-\lambda_{j}+j
\end{array}\right)\right]_{1 \leq i, j \leq \ell(\nu)},
$$

where $\mathrm{f}_{\nu / \lambda}$ is the number of elegant tableaux of shape $\nu / \lambda$ (see Def. 3.4).

Proof. Follows from the lattice path interpretation given in [Len00] (cf. [Yel17]) and the LindströmGessel-Viennot Lemma [GV89].

Proof of Theorem 4.5. By definition of $F_{\mu}$ it is clear that $F_{\mu} \in \Gamma$. Moreover, the expansion of $F_{\mu}$ in the basis $\left\{\tilde{G}_{\nu}\right\}$ contains only elements with $\ell(\nu) \leq \ell(\mu)$. To see this notice that since the Pieri rule (5) adds horizontal strips, we have that products $\tilde{G}_{\left(n_{1}\right)} \cdots \tilde{G}_{\left(n_{\ell}\right)}$ contains only elements $\tilde{G}_{\nu}$ with $\ell(\nu) \leq \ell$. Let $\ell=\ell(\mu)$. Lemma 4.7 gives

$$
F_{\mu}=\operatorname{det}\left[H_{0} \cdot h_{\mu_{i}-i+j}\right]_{1 \leq i, j \leq \ell}=\left(H_{0}\right)^{\ell} \operatorname{det}\left[h_{\mu_{i}-i+j}\right]_{1 \leq i, j \leq \ell}=\left(H_{0}\right)^{\ell} s_{\mu} .
$$

Using the following identity for Schur functions (which can be derived easily from Cauchy identities and LR expansions, or follows from skew Cauchy identities, see e.g. [Mac98, Ch. 1])

$$
s_{\mu}(\mathbf{x}) \prod_{i, j=1}^{\infty}\left(1+x_{i} y_{j}\right)=\sum_{\lambda \supset \mu} s_{\lambda}(\mathbf{x}) s_{\lambda^{\prime} / \mu^{\prime}}(\mathbf{y})
$$


for the specialization $\mathbf{y}=\left(1^{\ell}\right)$ and the Grothendieck expansion (6) we obtain

$$
\begin{aligned}
\left(H_{0}\right)^{\ell} s_{\mu}(\mathbf{x}) & =s_{\mu}(\mathbf{x}) \prod_{i=1}^{\infty}\left(1+x_{i}\right)^{\ell} \\
& =\sum_{\lambda \supset \mu} s_{\lambda}(\mathbf{x}) s_{\lambda^{\prime} / \mu^{\prime}}\left(1^{\ell}\right) \\
& =\sum_{\nu} \tilde{G}_{\nu}(\mathbf{x}) \sum_{\mu \subset \lambda \subset \nu}(-1)^{|\nu / \lambda|} \mathrm{f}_{\nu / \lambda} s_{\lambda^{\prime} / \mu^{\prime}}\left(1^{\ell}\right)
\end{aligned}
$$

where the sum is finite and contains only elements $\tilde{G}_{\nu}$ with $\ell(\nu) \leq \ell$. Let

$$
d(\nu, \mu):=\sum_{\mu \subset \lambda \subset \nu}(-1)^{|\nu / \lambda|} \mathrm{f}_{\nu / \lambda} s_{\lambda^{\prime} / \mu^{\prime}}\left(1^{\ell}\right)
$$

and we need to show that $d(\nu, \mu)=\mathrm{d}_{\nu / \mu}$ is the number of delegant tableaux.

From the Jacobi-Trudi identity we have (note that $\ell(\lambda) \leq \ell$ )

$$
s_{\lambda^{\prime} / \mu^{\prime}}\left(1^{\ell}\right)=\operatorname{det}\left[e_{\lambda_{i}-i-\mu_{j}+j}\left(1^{\ell}\right)\right]_{1 \leq i, j \leq \ell}=\operatorname{det}\left[\left(\begin{array}{c}
\ell \\
\lambda_{i}-i-\mu_{j}+j
\end{array}\right)\right]_{1 \leq i, j \leq \ell}
$$

Combining this determinantal formula, the formula in Lemma 4.9, the Cauchy-Binet identity (in such form indexed by partitions can be found in [Yel17]), and the formula in Lemma 4.8 we obtain

$$
\begin{aligned}
d(\nu, \mu) & =\sum_{\mu \subset \lambda \subset \nu}(-1)^{|\nu / \lambda|} \operatorname{det}\left[\left(\begin{array}{c}
\nu_{i}-\lambda_{j}+j-2 \\
\nu_{i}-i-\lambda_{j}+j
\end{array}\right)\right] \operatorname{det}\left[\left(\begin{array}{c}
\ell \\
\lambda_{i}-i-\mu_{j}+j
\end{array}\right)\right] \\
& =\sum_{\mu \subset \lambda \subset \nu} \operatorname{det}\left[(-1)^{\nu_{i}-i-\lambda_{j}+j}\left(\begin{array}{c}
\nu_{i}-\lambda_{j}+j-2 \\
\nu_{i}-i-\lambda_{j}+j
\end{array}\right)\right] \operatorname{det}\left[\left(\begin{array}{c}
\ell \\
\lambda_{i}-i-\mu_{j}+j
\end{array}\right)\right] \\
& =\operatorname{det}\left[\sum_{k}(-1)^{\nu_{i}-i-k}\left(\begin{array}{c}
\nu_{i}-k-2 \\
\nu_{i}-i-k
\end{array}\right)\left(\begin{array}{c}
\ell \\
k-\mu_{j}+j
\end{array}\right)\right] \\
& =\operatorname{det}\left[\left(\begin{array}{c}
\ell-i+1 \\
\nu_{i}-i-\mu_{j}+j
\end{array}\right)\right]_{1 \leq i, j \leq \ell} \\
& =\mathrm{d}_{\nu / \mu}
\end{aligned}
$$

Here we also used the identity

$$
\sum_{k}(-1)^{\nu_{i}-i-k}\left(\begin{array}{c}
\nu_{i}-k-2 \\
\nu_{i}-i-k
\end{array}\right)\left(\begin{array}{c}
\ell \\
k-\mu_{j}+j
\end{array}\right)=\left(\begin{array}{c}
\ell-i+1 \\
\nu_{i}-i-\mu_{j}+j
\end{array}\right)
$$

which can be derived by comparing the coefficients at $x^{\nu_{i}-i-\mu_{j}+j}$ from both sides of the generating function identity $(1+x)^{\ell-i+1}=(1+x)^{\ell}(1+x)^{-(i-1)}$.

Proof of Theorem 1.4. By Theorem 4.6 and the Edrei-Thoma theorem we have that the map

$$
h_{n} \longmapsto \frac{H_{n}(\varphi)}{H_{0}(\varphi)}=\frac{\tilde{G}_{(n)}(\varphi)+\tilde{G}_{(n+1)}(\varphi)}{1+\tilde{G}_{(1)}(\varphi)}, \quad n=1,2, \ldots
$$

defines a Schur-positive specialization of $\Lambda$. 
Lemma 4.10. Let $\varphi$ be a G-positive homomorphism of $\Gamma$. Suppose $\tilde{G}_{\mu}(\varphi)=0$ for some $\mu$. Then $\tilde{G}_{\lambda}(\varphi)=0$ for all $\lambda \supset \mu$.

Proof. From the Pieri rule we have

$$
0=\varphi\left(\tilde{G}_{(1)} \cdot \tilde{G}_{\mu}\right)=\sum_{\lambda / \mu \text { rook strip }} \tilde{G}_{\lambda}(\varphi) \geq 0
$$

Hence $\tilde{G}_{\lambda}(\varphi)=0$ for all $\lambda$ such that $\lambda / \mu$ is a rook strip. In particular, $\tilde{G}_{\lambda}(\varphi)=0$ for all $\lambda=\mu+\square$. Applying the same argument replacing $\mu$ with these partitions $\lambda$ further, we obtain that $\tilde{G}_{\lambda}(\varphi)=0$ for all $\lambda \supset \mu$.

Proof of Theorem 1.5. Since $\tilde{G}_{(1)}(\varphi)=1$, the map

$$
\rho: h_{n} \longmapsto \frac{1}{2}\left(\tilde{G}_{(n)}(\varphi)+\tilde{G}_{(n+1)}(\varphi)\right)
$$

defines a Schur-positive specialization by Theorem 1.4. Therefore,

$$
\rho(H(z))=1+\frac{1}{2} \sum_{n}\left(\tilde{G}_{(n)}(\varphi)+\tilde{G}_{(n+1)}(\varphi)\right) z^{n}=1+\sum_{n} \rho\left(h_{n}\right) z^{n}=e^{\gamma z} \prod_{n} \frac{1+\beta_{n} z}{1-\alpha_{n} z}
$$

for some nonnegative reals $\left\{\alpha_{n}\right\},\left\{\beta_{n}\right\}, \gamma$ such that $\sum_{n}\left(\alpha_{n}+\beta_{n}\right)<\infty$.

Note that from the Pieri rule

$$
\tilde{G}_{(n)} \cdot \tilde{G}_{(1)}=\tilde{G}_{(n+1)}+\tilde{G}_{(n, 1)}+\tilde{G}_{(n+1,1)}
$$

for all $n$ we obtain

$$
\tilde{G}_{(n+1)}(\varphi) \leq \tilde{G}_{(1)}(\varphi) \cdot \tilde{G}_{(n)}(\varphi)=\tilde{G}_{(n)}(\varphi) \leq \tilde{G}_{(1)}(\varphi)=1 .
$$

Consider two cases:

Case 1. If $\tilde{G}_{(2)}(\varphi)=1$. Then the identity $\left(\tilde{G}_{(1)}\right)^{2}=\tilde{G}_{(2)}+\tilde{G}_{\left(1^{2}\right)}+\tilde{G}_{(2,1)}$ implies that $\tilde{G}_{\left(1^{2}\right)}(\varphi)=0$ and hence by Lemma 4.10 we have $\tilde{G}_{\lambda}(\varphi)=0$ for all $\lambda \supset\left(1^{2}\right)$ (i.e. when $\lambda$ has at least two rows). From the identity (15) we obtain by induction that $\tilde{G}_{(n)}(\varphi)=1$ for all $n \geq 1$. Therefore, $\rho(H(z))=\frac{1}{1-z}$ which corresponds to the parameters $\gamma=0, \alpha_{1}=1, \alpha_{n+1}=0, \beta_{n}=0$ for all $n$. Conversely, we have the $G$-positive homomorphism $\tilde{G}_{\lambda}(\varphi)=\tilde{G}_{\lambda}(1)$ that meets these conditions.

Case 2. If $\tilde{G}_{(2)}(\varphi)=\delta<1$. From nonnegativity of $2 \times 2$ minors of the matrix $\left[\rho\left(h_{i-j}\right)\right]_{i, j \geq 0}$ (which is log-concavity) we have

$$
\frac{\rho\left(h_{n}\right)}{\rho\left(h_{n+1}\right)} \geq \frac{\rho\left(h_{n-1}\right)}{\rho\left(h_{n}\right)} \geq \ldots \geq \frac{1}{\rho\left(h_{1}\right)}=\frac{2}{1+\tilde{G}_{(2)}(\varphi)}=\frac{2}{1+\delta}>1 .
$$

Therefore, the radius of convergence of $\rho(H(z))$ is greater than 1. (In particular, $\alpha_{n}<1$.) Let now

$$
\rho(E(z)):=1+\sum_{n=1}^{\infty} \rho\left(e_{n}\right) z^{n}=\rho\left(H(-z)^{-1}\right)=e^{\gamma z} \prod_{n} \frac{1+\alpha_{n} z}{1-\beta_{n} z} .
$$

Similarly, we obtain that

$$
\frac{\rho\left(e_{n}\right)}{\rho\left(e_{n+1}\right)} \geq \frac{1}{\rho\left(e_{1}\right)}=\frac{1}{\rho\left(h_{1}\right)}=\frac{2}{1+\tilde{G}_{(2)}(\varphi)}=\frac{2}{1+\delta}>1
$$


and hence the radius of convergence of $\rho(E(z))$ is greater than 1 as well. In particular, $\beta_{n}<1$ for all $n$. Now we have

$$
\rho(H(-1))=\frac{1}{2}=e^{-\gamma} \prod_{n} \frac{1-\beta_{n}}{1+\alpha_{n}}<\infty
$$

and hence

$$
\gamma=\log 2-\sum_{n} \log \left(1+\alpha_{n}\right)+\sum_{n} \log \left(1-\beta_{n}\right)
$$

Let us show the converse part. Suppose $\rho$ is a Schur-positive specialization of $\Lambda$ with the parameters as above. Let us show that it extends to a $G$-positive specialization $\hat{\rho}$ such that

$$
\tilde{G}_{\lambda}(\hat{\rho}):=\sum_{\mu \supset \lambda} \mathrm{r}_{\mu / \lambda} \rho\left(s_{\mu}\right) \geq 0
$$

relying on Schur expansion of $\tilde{G}_{\lambda}$, see (6). We are going to show that the infinite sums

$$
0 \leq \sum_{\mu \supset \lambda} \mathrm{r}_{\mu / \lambda} \rho\left(s_{\mu}\right)<\infty
$$

converge. Let us check the normalization:

$$
\rho(H(-1))=1-\rho\left(h_{1}\right)+\rho\left(h_{2}\right)-\ldots=e^{-\gamma} \prod_{n} \frac{1-\beta_{n}}{1+\alpha_{n}}=\frac{1}{2}
$$

Hence,

$$
\tilde{G}_{(1)}(\hat{\rho})=-1+\frac{1}{\rho(H(-1))}=1 .
$$

Let $\lambda \vdash n$ and consider the expansion

$$
\left(\tilde{G}_{(1)}\right)^{n}-\tilde{G}_{\lambda}=\sum_{\nu} a_{\nu} \tilde{G}_{\nu} \in \Gamma_{+}
$$

Let also take the Schur expansion (in $\hat{\Lambda}$ )

$$
\left(\tilde{G}_{(1)}\right)^{n}=\sum_{\nu} b_{\nu} s_{\nu}, \quad b_{\nu} \in \mathbb{Z}_{\geq 0}
$$

Applying the specialization $\rho$ to these series we have

$$
\sum_{\nu} b_{\nu} \rho\left(s_{\nu}\right)=1
$$

converges. Finally observe that from (16)

$$
0 \leq \sum_{\mu \supset \lambda} \mathrm{r}_{\mu / \lambda} \rho\left(s_{\mu}\right) \leq \sum_{\nu} b_{\nu} \rho\left(s_{\nu}\right)=1 .
$$


4.1. Structure of $G$-positive specializations. Let us show that Schur-positive generators $\phi, \varepsilon, \pi$ (see subsec. 2.3) extend to $G$-positive specializations $\hat{\phi}, \hat{\varepsilon}, \hat{\pi}$.

Lemma 4.11. Let $\alpha, \beta, \gamma \in \mathbb{R}_{\geq 0}$ and $\beta<1$. Then the Schur-positive generators $\phi_{\alpha}, \varepsilon_{\beta}, \pi_{\gamma}$ of $\Lambda$ extend to $G$-positive specializations $\hat{\phi}_{\alpha}, \hat{\varepsilon}_{\beta}, \hat{\pi}_{\gamma}$ of $\Gamma$.

Proof. Let us check that

Indeed,

$$
\tilde{G}_{\lambda}\left(\hat{\phi}_{\alpha}\right)= \begin{cases}\alpha^{|\lambda|}, & \text { if } \ell(\lambda)=1 \\ 0, & \text { otherwise }\end{cases}
$$

$$
\sum_{\mu} \mathrm{r}_{\mu /(n)} \phi_{\alpha}\left(s_{\mu}\right)=\phi_{\alpha}\left(s_{(n)}\right)=\alpha^{n}
$$

as $\mathrm{r}_{\mu /(n)}=0$ if $\mu \supset(n)$ and $\mu \neq(n)$ and $\sum_{\mu \supset \lambda} \mathrm{r}_{\mu / \lambda} \phi_{\alpha}\left(s_{\mu}\right)=0$ if $\ell(\lambda) \geq 2$ since $\ell(\mu) \geq 2$ when $\mathrm{r}_{\mu / \lambda}>0$ and then $\phi_{\alpha}\left(s_{\mu}\right)=0$. Equivalently, $\hat{\phi}_{\alpha}$ just corresponds to the specialization $\left(x_{1}, x_{2}, \ldots\right) \mapsto(\alpha, 0,0, \ldots)$.

To see the effect of $\hat{\varepsilon}_{\beta}$, note that the involution $\omega: \hat{\Lambda} \rightarrow \hat{\Lambda}$ (extended on the completion $\hat{\Lambda}$ via $\left.\omega\left(s_{\lambda}\right)=s_{\lambda^{\prime}}\right)$ satisfies (see [PP16, Yel17])

$$
\omega\left(\tilde{G}_{\lambda}\left(x_{1}, x_{2}, \ldots\right)\right)=\tilde{G}_{\lambda^{\prime}}\left(\frac{x_{1}}{1-x_{1}}, \frac{x_{2}}{1-x_{2}}, \ldots\right) .
$$

Since $\varepsilon_{\beta}=\phi_{\beta} \circ \omega$ and $\hat{\varepsilon}_{\beta}=\hat{\phi}_{\beta} \circ \omega$ we obtain

$$
\tilde{G}_{\lambda}\left(\hat{\varepsilon}_{\beta}\right)= \begin{cases}\left(\frac{\beta}{1-\beta}\right)^{|\lambda|}, & \text { if } \lambda_{1}=1 \\ 0, & \text { otherwise. }\end{cases}
$$

As for the Plancherel specialization $\hat{\pi}_{\gamma}$ we have

$$
\sum_{\mu} \mathrm{r}_{\mu / \lambda} \pi_{\gamma}\left(s_{\mu}\right)=\sum_{\mu} \mathrm{r}_{\mu / \lambda} \frac{\gamma^{|\mu|} f^{\mu}}{|\mu| !} \in \mathbb{R}[[\gamma]]
$$

Let us show that this series converges for all $\gamma \in \mathbb{R}_{\geq 0}$ (and hence for $\gamma \in \mathbb{R}$ ). Note that for $\lambda=(1)$ we have:

$$
\sum_{\mu \supset(1)} \mathrm{r}_{\mu /(1)} \pi_{\gamma}\left(s_{\mu}\right)=\sum_{n \geq 1} \mathrm{r}_{\left(1^{n}\right) /(1)} \pi_{\gamma}\left(s_{\left(1^{n}\right)}\right)=\sum_{n=1}^{\infty} \frac{\gamma^{n}}{n !}=-1+e^{\gamma}
$$

which gives the value of $\tilde{G}_{(1)}\left(\hat{\pi}_{\gamma}\right)$. Consider the expansion

$$
\left(\tilde{G}_{(1)}\right)^{|\lambda|}=\sum_{\nu} a_{\nu} \tilde{G}_{\nu}=\sum_{\mu} b_{\mu} s_{\mu}, \quad a_{\nu}, b_{\mu} \geq 0
$$

Note that the function $\left(\tilde{G}_{(1)}\right)^{|\lambda|}-\tilde{G}_{\lambda}$ is both Grothendieck and Schur positive and hence we have $b_{\mu} \geq \mathrm{r}_{\mu / \lambda}$ implying

$$
0 \leq \sum_{\mu} \mathrm{r}_{\mu / \lambda} \pi_{\gamma}\left(s_{\mu}\right) \leq \sum_{\mu} b_{\mu} \pi_{\gamma}\left(s_{\mu}\right)=\left(-1+e^{\gamma}\right)^{|\lambda|}
$$

Therefore, the series

$$
\tilde{G}_{\lambda}\left(\hat{\pi}_{\gamma}\right)=\sum_{\mu} \mathrm{r}_{\mu / \lambda} \pi_{\gamma}\left(s_{\mu}\right)
$$

converges for all $\gamma \in \mathbb{R}_{\geq 0}$ and the homomorphism $\hat{\pi}_{\gamma}$ is a well-defined and $G$-positive. 
Proposition 4.12. Let $\varphi$ be the union of extended Schur-positive generators $\left(\hat{\phi}_{\alpha_{n}}\right),\left(\hat{\varepsilon}_{\beta_{n}}\right)$, $\hat{\pi}_{\gamma}$ for nonnegative real parameters $\left\{\alpha_{n}\right\},\left\{\beta_{n}<1\right\}, \gamma$, such that $\sum_{n}\left(\alpha_{n}+\beta_{n}\right)<\infty$. Then $\varphi$ is $G$-positive. In particular, we have

$$
\tilde{G}_{(1)}(\varphi)=-1+e^{\gamma} \prod_{n=1}^{\infty} \frac{1+\alpha_{n}}{1-\beta_{n}}
$$

and the following generating function for the elements $\left\{\tilde{G}_{(n)}(\varphi)\right\}$

$$
1+(z+1) \sum_{n=1}^{\infty} \tilde{G}_{(n)}(\varphi) z^{n-1}=e^{\gamma(z+1)} \prod_{n=1}^{\infty} \frac{1+\alpha_{n}}{1-\alpha_{n} z} \prod_{n=1}^{\infty} \frac{1+\beta_{n} z}{1-\beta_{n}}
$$

Proof. By Lemma 4.11, the specializations $\hat{\phi}_{\alpha_{n}}, \hat{\varepsilon}_{\beta_{n}}, \hat{\pi}_{\gamma}$ are all $G$-positive and hence their union is also $G$-positive given $\tilde{G}_{(1)}(\varphi)$ converges. The formulas (17), (18) then follow from Schur expansions of $\left\{\tilde{G}_{(n)}\right\}$, see Lemma 4.7 .

Remark 4.13. Instead of the $G$-positive generators $\hat{\varepsilon}_{\beta^{\prime}}$ for $\beta^{\prime}<1$ we can use another specializations $\psi_{\beta}:=\hat{\phi}_{\beta} \circ \tau$, i.e. $\psi_{\beta}: \tilde{G}_{\lambda} \mapsto \tilde{G}_{\lambda^{\prime}}\left(\hat{\phi}_{\beta}\right)$ for $\beta \geq 0$. Here we have $\tilde{G}_{\lambda}\left(\psi_{\beta}\right)=\beta^{|\lambda|} \geq 0$, if $\lambda_{1}=1$ and 0 otherwise. One can see that $\psi_{\beta}=\hat{\varepsilon}_{\beta^{\prime}}$, where $\beta^{\prime}=\beta /(1+\beta)$. Then we can alternatively redefine $G$-positive homomorphisms $\varphi$ in Theorem 4.12 as the union of generators $\hat{\phi}_{\alpha_{n}}, \psi_{\beta_{n}}$, and $\hat{\pi}_{\gamma}$ for nonnegative reals $\left\{\alpha_{n}\right\},\left\{\beta_{n}\right\}, \gamma$ such that $\sum_{n}\left(\alpha_{n}+\beta_{n}\right)<\infty$. In particular,

$$
\tilde{G}_{(1)}(\varphi)=-1+e^{\gamma} \prod_{n=1}^{\infty}\left(1+\alpha_{n}\right)\left(1+\beta_{n}\right) .
$$

\section{HaRmonic Functions AND Boundary of A FILTEREd Young's GRAPH}

Consider the (infinite) filtered Young graph $\widetilde{\mathbb{Y}}$ defined as follows:

(i) its vertices are labeled by partitions $\lambda$;

(ii) there is an $\operatorname{arc} \lambda \rightarrow \mu$ iff $\mu / \lambda$ is a rook strip (i.e. no two boxes lie in the same row or column)

Definition 5.1. Say that a function $\varphi: \mathcal{P} \rightarrow \mathbb{R}_{\geq 0}$ is harmonic on $\widetilde{\mathbb{Y}}$ if

$$
\varphi(\varnothing)=1 \quad \text { and } \quad \varphi(\lambda)=\sum_{\lambda \rightarrow \mu} \varphi(\mu)
$$

Let $H(\widetilde{\mathbb{Y}})$ be the set of harmonic functions on $\widetilde{\mathbb{Y}}$. Then $H(\widetilde{\mathbb{Y}})$ is a convex set and let $\partial \widetilde{\mathbb{Y}}$ be the set of extreme points of $H(\widetilde{\mathbb{Y}})$, or the boundary of $\widetilde{\mathbb{Y}}$.

For every function $\varphi \in H(\widetilde{\mathbb{Y}})$ define the linear functional $\hat{\varphi}: \Gamma \rightarrow \mathbb{R}$ such that

$$
\hat{\varphi}\left(\tilde{G}_{\lambda}\right):=\varphi(\lambda) .
$$

Alternatively, consider linear functionals $\hat{\varphi}: \Gamma \rightarrow \mathbb{R}$ satisfying the following properties:

(i) $\hat{\varphi}(1)=1$

(ii) $\hat{\varphi}\left(\tilde{G}_{\lambda}\right) \geq 0$

(iii) $\hat{\varphi}\left(\tilde{G}_{(1)} \cdot \tilde{G}_{\lambda}\right)=\hat{\varphi}\left(\tilde{G}_{\lambda}\right)$ 
Using the simple Pieri rule we obtain

$$
\hat{\varphi}\left(\tilde{G}_{\lambda}\right)=\hat{\varphi}\left(\tilde{G}_{(1)} \cdot \tilde{G}_{\lambda}\right)=\sum_{\mu / \lambda \text { rook strip }} \hat{\varphi}\left(\tilde{G}_{\mu}\right)
$$

i.e. the function $\varphi(\lambda)=\hat{\varphi}\left(\tilde{G}_{\lambda}\right)$ is harmonic on $\widetilde{\mathbb{Y}}$.

The next theorem is a version of the Vershik-Kerov "ring theorem" [KV80] for $\widetilde{\mathbb{Y}}$.

Theorem 5.2. We have: $\varphi \in \partial \widetilde{\mathbb{Y}}$ i.e. $\varphi$ is extreme if and only if the linear functional $\hat{\varphi}$ is a normalized $G$-positive homomorphism of $\Gamma$.

Proof. The same proof as in the case of graded Young graph and the ring $\Lambda$ with the Schur basis (see [BO17], also [GO06] for a general statement) works in our case as $\tilde{G}_{\mu} \tilde{G}_{\nu} \in \Gamma_{+}$and $\left(\tilde{G}_{(1)}\right)^{|\lambda|}-\tilde{G}_{\lambda} \in \Gamma_{+}$for all $\lambda, \mu, \nu$. We reproduce it here for completeness.

Suppose $f \in \Gamma_{+}$such that $\hat{\varphi}(f)>0$. Let us then check that the new function

$$
\varphi_{f}(g):=\frac{\hat{\varphi}(f g)}{\hat{\varphi}(f)}, \quad g \in \Gamma
$$

also satisfies the properties (i)-(iii) of linear functionals $\hat{\varphi}$. Indeed, we have (i) $\varphi_{f}(1)=1$, (ii) $\varphi_{f}\left(\tilde{G}_{\lambda}\right)=\hat{\varphi}\left(f \cdot \tilde{G}_{\lambda}\right) / \hat{\varphi}(f) \geq 0$ since $f \cdot \tilde{G}_{\lambda} \in \Gamma_{+}$expands as a nonnegative linear combination of $\left\{\tilde{G}_{\mu}\right\}$, and for (iii) we have

$$
\varphi_{f}\left(\tilde{G}_{(1)} \cdot \tilde{G}_{\lambda}\right)=\hat{\varphi}\left(f \cdot \tilde{G}_{(1)} \cdot \tilde{G}_{\lambda}\right) / \hat{\varphi}(f)=\hat{\varphi}\left(f \cdot \tilde{G}_{\lambda}\right) / \hat{\varphi}(f)=\varphi_{f}\left(\tilde{G}_{\lambda}\right) .
$$

Suppose that $\varphi$ is extreme. We need to show that $\hat{\varphi}\left(\tilde{G}_{\mu} \cdot G\right)=\hat{\varphi}\left(\tilde{G}_{\mu}\right) \hat{\varphi}(G)$ for all $\mu \in \mathcal{P}$ and $G \in \Gamma$. Note that for $\lambda \vdash n$ we have $\left(\tilde{G}_{(1)}\right)^{n}-\tilde{G}_{\lambda} \in \Gamma_{+}$as a consequence of Pieri rule.

If $\hat{\varphi}\left(\tilde{G}_{\mu}\right)=0$, then for $\lambda \vdash n$ we have

$$
0 \leq \hat{\varphi}\left(\tilde{G}_{\mu} \cdot \tilde{G}_{\lambda}\right) \leq \hat{\varphi}\left(\tilde{G}_{\mu} \cdot\left(\tilde{G}_{(1)}\right)^{n}\right)=\hat{\varphi}\left(\tilde{G}_{\mu}\right)=0 .
$$

Hence $\hat{\varphi}\left(\tilde{G}_{\mu} \cdot \tilde{G}_{\lambda}\right)=0$ and the multiplicativity holds.

If $\hat{\varphi}\left(\tilde{G}_{\mu}\right)>0$ for $\mu \vdash m$, consider the functions

$$
g_{1}:=\frac{1}{2} \tilde{G}_{\mu}, \quad g_{2}:=\left(\tilde{G}_{(1)}\right)^{m}-g_{1} \in \Gamma_{+} .
$$

Then for $G \in \Gamma$ we have

$$
\hat{\varphi}(G)=\hat{\varphi}\left(\left(\tilde{G}_{(1)}\right)^{m} \cdot G\right)=\hat{\varphi}\left(g_{1} \cdot G\right)+\hat{\varphi}\left(g_{2} \cdot G\right) .
$$

We clearly have $c_{1}:=\hat{\varphi}\left(g_{1}\right)>0, c_{2}:=\hat{\varphi}\left(g_{2}\right)>0$ such that $c_{1}+c_{2}=1$ and hence

$$
\hat{\varphi}=c_{1} \varphi_{g_{1}}+c_{2} \varphi_{g_{2}} .
$$

Since $\varphi$ is extreme we have $\hat{\varphi}=\varphi_{g_{1}}$ which implies that $\hat{\varphi}(G)=\hat{\varphi}\left(\tilde{G}_{\mu} \cdot G\right) / \hat{\varphi}\left(\tilde{G}_{\mu}\right)$ as needed.

To show the converse part, suppose that $\hat{\varphi}$ is a homomorphism of $\Gamma$, and let us show that $\varphi$ is extreme. By Choquet's theorem we have the integral presentation

$$
\hat{\varphi}(G)=\int_{f \in \partial \widetilde{\mathbb{Y}}} \hat{f}(G) \mu(d f), \quad G \in \Gamma
$$


where $\mu$ is a probability measure on $\partial \widetilde{\mathbb{Y}}$. View the function $f \rightarrow \hat{f}(G)$ as a random variable on the space $(\partial \widetilde{\mathbb{Y}}, \mu)$. By the integral representation its expectation is $\hat{\varphi}(G)$. We also have $\hat{f}$ is a homomorphism of $\Gamma$ by the argument above. Now observe that

$$
\left(\int_{f \in \partial \widetilde{\mathbb{Y}}} \hat{f}(G) \mu(d f)\right)^{2}=\hat{\varphi}(G)^{2}=\hat{\varphi}\left(G^{2}\right)=\int_{f \in \partial \widetilde{\mathbb{Y}}} \hat{f}\left(G^{2}\right) \mu(d f)=\int_{f \in \partial \widetilde{\mathbb{Y}}} \hat{f}(G)^{2} \mu(d f)
$$

implying that $\hat{f}(G)$ has variance 0 . Therefore, $\hat{f}(G)=\hat{\varphi}(G)$ almost surely. Hence $\mu$ is a deltameasure and $\varphi$ is extreme.

\section{Positivity of Grothendieck polynomials with alternating signs}

Let us define the functions

$$
G_{\lambda / / \mu}(\mathbf{x}):=(-1)^{|\lambda / \mu|} \tilde{G}_{\lambda / / \mu}(-\mathbf{x})
$$

which is a usual definition of symmetric Grothendieck polynomials whose monomial expansion has alternating signs. Namely, we have (see [Yel19])

$$
G_{\lambda / / \mu}(\mathbf{x})=\sum_{T \in S S V T(\lambda / / \mu)}(-1)^{|T|-|\lambda / \mu|} x^{T}
$$

where $|T|$ is the number of entries in $T$. Clearly, $\left\{G_{\lambda}\right\}$ is a basis of $\Gamma$ and we have

$$
G_{\mu} \cdot G_{\nu}=\sum_{\lambda}(-1)^{|\lambda|-|\mu|-|\nu|} c_{\mu \nu}^{\lambda} G_{\lambda}
$$

Note also that

$$
G_{(1) / /(1)}=1-G_{(1)}=\prod_{n=1}^{\infty}\left(1-x_{n}\right) .
$$

Definition 6.1. We say that a homomorphism $\varphi: \Gamma \rightarrow \mathbb{R}$ is $\bar{G}$-positive if $\varphi\left(G_{\lambda / / \mu}\right) \geq 0$ for all $\lambda, \mu$.

Lemma 6.2. Let $\varphi_{1}, \varphi_{2}$ be $\bar{G}$-positive homomorphisms of $\Gamma$. Then their union $\varphi=\left(\varphi_{1}, \varphi_{2}\right)$ is also $\bar{G}$-positive.

Proof. Follows from the branching formula (3) which remains the same for the functions $G$.

Lemma 6.3. Let $\varphi$ be a $\bar{G}$-positive homomorphism of $\Gamma$. Then

$$
1 \geq G_{(1)}(\varphi) \geq G_{(2)}(\varphi) \geq \ldots \geq 0
$$

Proof. We have for all $n \geq 0$

$$
G_{(n)}(\varphi)-G_{(n+1)}(\varphi)=G_{(n+1) / /(1)}(\varphi) \geq 0 .
$$

Note also that $G_{(0)}(\varphi)=G_{\varnothing}(\varphi)=1$.

Theorem 6.4. Let $\varphi$ be a $\bar{G}$-positive homomorphism of $\Gamma$ and suppose $G_{(1)}(\varphi) \neq 1$. Then

$$
\rho: h_{n} \longmapsto \frac{G_{(n)}(\varphi)-G_{(n+1)}(\varphi)}{1-G_{(1)}(\varphi)}
$$

defines a Schur-positive specialization of $\Lambda$ with nonnegative parameters $\left\{\alpha_{n}\right\},\left\{\beta_{n}\right\}, \gamma$ such that

$$
G_{(1)}(\varphi)=1-e^{-\gamma} \prod_{n=1}^{\infty} \frac{1-\alpha_{n}}{1+\beta_{n}} \in[0,1)
$$


Proof. Suppose $\varphi$ is a $\bar{G}$-positive homomorphism of $\Gamma$ with $G_{(1)}(\varphi) \neq 1$. By Lemma 6.3 we get

$$
1>G_{(1)}(\varphi) \geq G_{(2)}(\varphi) \geq \ldots \geq 0
$$

Recall that we have the following infinite positive expansion of $s_{\lambda}$ in the 'basis' $\left\{G_{\mu}\right\}$ which follows from (6) (see [Len00]):

$$
s_{\lambda}=\sum_{\mu \supset \lambda} \mathrm{f}_{\mu / \lambda} G_{\mu}
$$

where $f_{\mu / \lambda}$ is the number of elegant tableaux of shape $\mu / \lambda$ (see Def. 3.4). Therefore, if for all $\lambda$

$$
0 \leq \sum_{\mu \supset \lambda} \mathrm{f}_{\mu / \lambda} G_{\mu}(\varphi)<\infty
$$

then we can apply the homomorphism $\varphi$ to define $\rho=\rho(\varphi): \Lambda \rightarrow \mathbb{R}$ by letting

$$
\rho\left(s_{\lambda}\right):=\sum_{\mu \supset \lambda} \mathrm{f}_{\mu / \lambda} G_{\mu}(\varphi)
$$

which becomes a well-defined Schur-positive specialization. Let us consider the identity (follows by Lemma 4.7)

$$
h_{1}=\frac{G_{(1)}-G_{(2)}}{1-G_{(1)}}=\sum_{\mu} \mathrm{f}_{\mu /(1)} G_{\mu}
$$

and the expansion

$$
\left(h_{1}\right)^{n}=\left(\frac{G_{(1)}-G_{(2)}}{1-G_{(1)}}\right)^{n}=\sum_{\mu} a_{\mu} G_{\mu}, \quad a_{\mu} \geq 0 .
$$

Since the function $\left(h_{1}\right)^{n}-s_{\lambda}$ is Schur-positive, we must have $a_{\mu} \geq \mathrm{f}_{\mu / \lambda}$ for all $\mu$. Applying the homomorphism $\varphi$ to (23) we obtain that the series

$$
\sum_{\mu} a_{\mu} G_{\mu}(\varphi)=\left(\frac{G_{(1)}(\varphi)-G_{(2)}(\varphi)}{1-G_{(1)}(\varphi)}\right)^{n}
$$

converges since $G_{(1)}(\varphi)<1$. Therefore,

$$
0 \leq \sum_{\mu \supset \lambda} \mathrm{f}_{\mu / \lambda} G_{\mu}(\varphi) \leq \sum_{\mu} a_{\mu} G_{\mu}(\varphi)<\infty
$$

as needed. So we have defined a Schur-positive specialization given by (22). Let us check its values on the generators $h_{n}$ :

$$
\rho\left(h_{n}\right)=\sum_{\mu \supset(n)} \mathrm{f}_{\mu /(n)} G_{\mu}(\varphi)=\frac{G_{(n)}(\varphi)-G_{(n+1)}(\varphi)}{1-G_{1}(\varphi)}
$$

where the last identity makes sense as it is an identity for $\left\{G_{\mu}\right\}$ (see Lemma 4.7) and $G_{1}(\varphi)<1$.

Let us now show that the series

$$
\rho(H(1))=1+\sum_{n=1}^{\infty} \rho\left(h_{n}\right)
$$


converges. By $(20)$ there exists $\lim _{N \rightarrow \infty} G_{(N)}(\varphi)=A \in[0,1]$. Using this and (24) we have

$$
\rho(H(1))=\lim _{N \rightarrow \infty}\left(1+\sum_{n=1}^{N} \rho\left(h_{n}\right)\right)=\lim _{N \rightarrow \infty} \frac{1-G_{(N+1)}(\varphi)}{1-G_{1}(\varphi)}=\frac{1-A}{1-G_{1}(\varphi)}
$$

On the other hand, using the identity

$$
H(1)=\frac{1}{1-G_{(1)}}=\sum_{k=0}^{\infty}\left(G_{(1)}\right)^{k}
$$

we obtain

$$
\rho(H(1))=\frac{1}{1-G_{(1)}(\varphi)}
$$

Furthermore, since $\rho$ is Schur-positive, it is parametrized by some nonnegative reals $\left\{\alpha_{n}\right\},\left\{\beta_{n}\right\}, \gamma$, and we have

or

$$
1 \leq \rho(H(1))=\frac{1}{1-G_{(1)}(\varphi)}=e^{\gamma} \prod_{n=1}^{\infty} \frac{1+\beta_{n}}{1-\alpha_{n}}<\infty
$$

$$
G_{(1)}(\varphi)=1-e^{-\gamma} \prod_{n=1}^{\infty} \frac{1-\alpha_{n}}{1+\beta_{n}} \in[0,1)
$$

as needed.

\subsection{Structure of $\bar{G}$-positive specializations.}

Lemma 6.5. Let $\alpha, \beta, \gamma \in \mathbb{R}_{\geq 0}$ and $\alpha \leq 1$. Then the Schur-positive generators of $\Lambda$ extend to $\bar{G}$-positive specializations $\hat{\phi}_{\alpha}, \hat{\varepsilon}_{\beta}$, $\hat{\pi}_{\gamma}$ of $\Gamma$.

Proof. First, using the single variable substitution (4) we have

$$
G_{\lambda / / \mu}\left(\hat{\phi}_{\alpha}\right)=\alpha^{|\lambda / \mu|}(1-\alpha)^{a(\lambda / / \mu)} \geq 0 .
$$

Next, for the specialization $\hat{\varepsilon}_{\beta}$ we have

$$
G_{\lambda / / \mu}\left(\hat{\varepsilon}_{\beta}\right)=G_{\lambda^{\prime} / / \mu^{\prime}}\left(\hat{\phi}_{\beta^{\prime}}\right)=\beta^{\left|\lambda^{\prime} / \mu^{\prime}\right|} /(1+\beta)^{\left|\lambda^{\prime} / \mu^{\prime}\right|+a\left(\lambda^{\prime} / / \mu^{\prime}\right)} \geq 0, \text { where } \beta^{\prime}=\beta /(1+\beta) .
$$

Here we used the formula (see [Yel17])

$$
\omega\left(G_{\lambda / / \mu}\left(x_{1}, x_{2}, \ldots\right)\right)=G_{\lambda^{\prime} / / \mu^{\prime}}\left(x_{1} /\left(1+x_{1}\right), x_{2} /\left(1+x_{2}\right), \ldots\right) .
$$

For the Plancherel specialization $\hat{\pi}_{\gamma}$, let us first show that the power series $G_{\lambda / / \mu}\left(\hat{\pi}_{\gamma}\right) \in \mathbb{R}[[\gamma]]$ converges for all $\gamma \in \mathbb{R}$. From the definition of $G$ functions we have

$$
G_{\lambda / / \mu}\left(\hat{\pi}_{\gamma}\right)=(-1)^{|\lambda / \mu|} \tilde{G}_{\lambda / / \mu}\left(\hat{\pi}_{-\gamma}\right)
$$

and since $\tilde{G}_{\lambda / / \mu}\left(\hat{\pi}_{-\gamma}\right) \in \mathbb{R}[[\gamma]]$ converges for all $\gamma \in \mathbb{R}$ (see proof of Lemma 4.11), the same holds for $G_{\lambda / / \mu}\left(\hat{\pi}_{\gamma}\right)$. To see nonnegativity, notice that $\pi_{\gamma}$ can be realized as follows:

$$
G_{\lambda / / \mu}\left(\hat{\pi}_{\gamma}\right)=\lim _{N \rightarrow \infty} G_{\lambda / / \mu}(\underbrace{\gamma / N, \ldots, \gamma / N}_{N \text { times }})=\lim _{N \rightarrow \infty} G_{\lambda / / \mu}(\underbrace{\hat{\phi}_{\gamma / N}, \ldots, \hat{\phi}_{\gamma / N}}_{N \text { times }})
$$

for $\gamma / N \leq 1$ we have $\hat{\phi}_{\gamma / N}$ is $\bar{G}$-positive and so the union $\left(\hat{\phi}_{\gamma / N}, \ldots, \hat{\phi}_{\gamma / N}\right)$ is $\bar{G}$-positive as well. Hence $G_{\lambda / / \mu}\left(\hat{\pi}_{\gamma}\right) \geq 0$ as desired. 
Proposition 6.6. Let $\rho$ be a Schur-positive specialization of $\Lambda$ with nonnegative real parameters $\left\{\alpha_{n}<1\right\},\left\{\beta_{n}\right\}, \gamma$ such that

$$
\delta:=1-e^{-\gamma} \prod_{n=1}^{\infty} \frac{1-\alpha_{n}}{1+\beta_{n}} \in[0,1)
$$

Then it extends to a $\bar{G}$-positive homomorphism $\hat{\rho}$ of $\Gamma$ that is a union of Schur-positive generators $\hat{\pi}_{\gamma},\left(\hat{\phi}_{\alpha_{n}}\right),\left(\hat{\varepsilon}_{\beta_{n}}\right)$. In particular, $G_{(1)}(\hat{\rho})=\delta$ and the generating function for the elements $\left\{G_{(n)}(\hat{\rho})\right\}$ is the following

$$
1+(z-1) \sum_{n=1}^{\infty} G_{(n)}(\hat{\rho}) z^{n-1}=e^{\gamma(z-1)} \prod_{n=1}^{\infty} \frac{1-\alpha_{n}}{1-\alpha_{n} z} \prod_{n=1}^{\infty} \frac{1+\beta_{n} z}{1+\beta_{n}} .
$$

Proof. By the factorization form in Theorem 2.4, $\rho$ as a union of Schur-positive generators $\pi_{\gamma},\left(\phi_{\alpha_{n}}\right)$, $\left(\varepsilon_{\beta_{n}}\right)$ (see subsec. 2.3). Then these Schur-positive generators extend to $\bar{G}$-positive specializations by Lemma 6.5. Define $\varphi$ as the union of positive generators $\pi_{\gamma},\left(\hat{\phi}_{\alpha_{n}}\right),\left(\varepsilon_{\beta_{n}}\right)$. Then the formulas (25), (26) follow by Schur expansions of $\left\{G_{n}\right\}$, see Lemma 4.7.

6.2. The normalized case $G_{(1)}(\varphi)=1$. Recall that $G_{(1)}=1-\prod_{n}\left(1-x_{n}\right)$ and hence a normalized homomorphism of $\Gamma$ maps $\prod_{n}\left(1-x_{n}\right)$ to 0 .

Lemma 6.7. We have $G_{\lambda / / \mu}(1, \mathbf{x})=G_{\tilde{\lambda} / / \mu}(\mathbf{x})$, where $\tilde{\lambda}=\left(\lambda_{2}, \lambda_{3}, \ldots\right)$, i.e. the first row in $\lambda$ is removed.

Proof. By the branching formula (3) we have $G_{\lambda / / \mu}(1, \mathbf{x})=\sum_{\nu} G_{\lambda / / \nu}(1) G_{\nu / / \mu}(\mathbf{x})$. From the single variable formula (4) it is not hard to see that we have for $\nu \neq \varnothing$

$$
G_{\lambda / / \nu}(1)= \begin{cases}1, & \text { if } \nu=\tilde{\lambda} \\ 0, & \text { otherwise }\end{cases}
$$

which implies the needed.

Theorem 6.8. Let $\varphi: \Gamma \rightarrow \mathbb{R}$ be a homomorphism and $\varphi^{\prime}=(1, \varphi)$ (where $\left.1=\hat{\phi}_{1}\right)$. Then:

(i) $\varphi^{\prime}$ is a normalized homomorphism of $\Gamma$.

(ii) $\varphi$ is $\bar{G}$-positive if and only if $\varphi^{\prime}$ is $\bar{G}$-positive.

Proof. (i) $\varphi^{\prime}$ is normalized since $G_{(1)}\left(1, \varphi^{\prime}\right)=1-(1-1) G_{(1)}(\varphi)=1$.

(ii) If $\varphi$ is $\bar{G}$-positive, then $\varphi^{\prime}$ is also $\bar{G}$-positive since it is a union of $\bar{G}$-positive specializations $\mathbf{x} \mapsto(1,0,0, \ldots)$ and $\varphi$.

Conversely, suppose $\varphi^{\prime}$ is $\bar{G}$-positive. By Lemma 6.7 we obtain $0 \leq G_{\lambda / / \mu}\left(\varphi^{\prime}\right)=G_{\lambda / / \mu}(1, \varphi)=$ $G_{\tilde{\lambda} / / \mu}(\varphi)$ implying that $\varphi$ is $\bar{G}$-positive.

Remark 6.9. Lemma 6.7 is a slight extension of Buch's observation [Buc02] for $\mu=\varnothing$.

Remark 6.10. Using these properties we also obtain the following $\bar{G}$-positive specializations:

(i) $\mathbf{x} \mapsto 1^{N}$ for which $G_{\lambda}\left(1^{N}\right)=1$ if $\ell(\lambda) \leq N$ and 0 otherwise;

(ii) by letting $N \rightarrow \infty$, we get $G_{\lambda} \mapsto 1$; in other words, the linear map $\varphi: \Gamma \rightarrow \mathbb{R}$ given by $\varphi: G_{\lambda} \mapsto 1$ for all $\lambda$ is a well-defined $\bar{G}$-positive homomorphism of $\Gamma$.

This implies certain identities for dual families given in [Yel19b] from combinatorial perspective. 


\section{Dual Grothendieck-Positive specializations}

Definition 7.1. A reverse plane partition (RPP) of shape $\lambda / \mu$ is a filling of its boxes with positive integers that weakly increase both in rows from left to right and in columns from top to bottom. Let $R P P(\lambda / \mu)$ be the set of RPP of shape $\lambda / \mu$. For $T \in R P P(\lambda / \mu)$, define the monomial $x^{T}=$ $\prod_{i \geq 1} x_{i}^{c_{i}}$, where $c_{i}$ is the number of columns that contain $i$. Recall also that $c(\lambda / \mu)$ denotes the number of columns of $\lambda / \mu$.

Definition 7.2 ([LP07]). The dual symmetric Grothendieck polynomials $g_{\lambda / \mu}$ are defined as follows:

$$
g_{\lambda / \mu}=g_{\lambda / \mu}\left(x_{1}, x_{2}, \ldots\right):=\sum_{T \in R P P(\lambda / \mu)} x^{T}
$$

Note that $g_{\lambda}=s_{\lambda}+\{$ lower degree elements $\}$ since the largest weight monomials from RPP correspond to SSYT of shape $\lambda$, and hence $\left\{g_{\lambda}\right\}$ is a basis of $\Lambda$. The basis $\left\{g_{\lambda}\right\}$ is in fact dual to $\left\{G_{\lambda}\right\}$ via the Hall inner product for which Schur functions form an orthonormal basis.

Similarly as for $G_{\lambda}$, there is an involutive automorphism $\hat{\tau}: \Lambda \rightarrow \Lambda$ given on generators by

$$
\hat{\tau}: h_{n} \longmapsto \sum_{i=1}^{n}\left(\begin{array}{c}
n-1 \\
i-1
\end{array}\right) e_{i}
$$

and for which [Yel19]

$$
\hat{\tau}\left(g_{\lambda / \mu}\right)=g_{\lambda^{\prime} / \mu^{\prime}}
$$

Proposition 7.3 ([Yel19]). The following branching formula holds

$$
g_{\lambda / \mu}(\mathbf{x}, \mathbf{y})=\sum_{\mu \subset \nu \subset \lambda} g_{\lambda / \nu}(\mathbf{x}) g_{\nu / \mu}(\mathbf{y})
$$

For a single variable $x$ we have

$$
g_{\lambda / \mu}(x)= \begin{cases}x^{c(\lambda / \mu)}, & \text { if } \mu \subset \lambda \\ 0, & \text { otherwise. }\end{cases}
$$

Definition 7.4 (Dual Grothendieck-positive specializations). A homomorphism $\rho: \Lambda \rightarrow \mathbb{R}$ is called g-positive if $\rho\left(g_{\lambda / \mu}\right) \geq 0$ for all $\lambda, \mu$.

We describe a class of $g$-positive specializations as follows:

Proposition 7.5. Let $\rho: \Lambda \rightarrow \mathbb{R}$ be a specialization given by

$$
\rho(H(z))=1+\sum_{n=1}^{\infty} \rho\left(h_{n}\right) z^{n}=e^{\gamma z+\delta z /(1-z)} \prod_{n=1}^{\infty} \frac{1}{1-\alpha_{n} z} \prod_{n=1}^{\infty}\left(1+\frac{\beta_{n} z}{1-z}\right)
$$

for nonnegative reals $\left\{\alpha_{n}\right\},\left\{\beta_{n}\right\}, \gamma, \delta$ such that $\sum_{n}\left(\alpha_{n}+\beta_{n}\right)<\infty$. Then $\rho$ is g-positive.

Proof. First, note that $g_{(n)}=h_{n}$. By the branching formula (27), we obtain that union of $g$-positive specializations is also $g$-positive. Let $\alpha, \beta, \gamma, \delta \geq 0$. The following specializations are all $g$-positive:

(a) $\phi_{\alpha}:\left(x_{1}, x_{2}, \ldots\right) \mapsto(\alpha, 0,0, \ldots)$. Here $\phi_{\alpha}\left(g_{\lambda / \mu}\right)=\alpha^{c(\lambda / \mu)} \geq 0$ and we have

$$
\phi_{\alpha}\left(h_{n}\right)=\alpha^{n}, \quad \phi_{\alpha}(H(z))=\frac{1}{1-\alpha z}
$$


(b) $\psi_{\beta}:=\phi_{\beta} \circ \hat{\tau}$. Here $\psi_{\beta}\left(g_{\lambda / \mu}\right)=\beta^{c\left(\lambda^{\prime} / \mu^{\prime}\right)} \geq 0$ and we have

$$
\psi_{\beta}\left(h_{n}\right)=\beta, \quad \psi_{\beta}(H(z))=1+\frac{\beta z}{1-z}
$$

(c) The Plancherel specialization $\pi_{\gamma}: p_{1} \mapsto \gamma$ and $p_{k} \mapsto 0$ for $k \geq 2$. Since the polynomials $g_{\lambda / \mu}$ are Schur-positive (see [Gal17], also [LP07] for straight shapes), we have $\pi_{\gamma}\left(g_{\lambda / \mu}\right) \geq 0$ and here

$$
\pi_{\gamma}(H(z))=e^{\gamma z}
$$

(d) The dual Plancherel specialization $\tilde{\pi}_{\delta}:=\pi_{\delta} \circ \hat{\tau}$. Here $\tilde{\pi}_{\delta}\left(g_{\lambda / \mu}\right)=\pi_{\delta}\left(g_{\lambda^{\prime} / \mu^{\prime}}\right) \geq 0$ and we have

$$
\tilde{\pi}_{\delta}(H(z))=e^{\delta z /(1-z)} .
$$

Now we can define the specialization $\rho$ as the union of specializations $\pi_{\gamma}, \tilde{\pi}_{\delta},\left(\phi_{\alpha_{n}}\right),\left(\psi_{\beta_{n}}\right)$ which gives $g$-positivity.

We conjecture that the converse is also true.

Conjecture 7.6. Every $g$-positive specialization is characterized by (29).

Remark 7.7. Equivalently, $g$-positive specializations $\rho$ in Prop. 7.5 are given by

$$
\begin{aligned}
& \rho: p_{1} \longmapsto \gamma+\delta+\sum_{n}\left(\alpha_{n}+\beta_{n}\right) \\
& \rho: p_{k} \longmapsto \delta k+\sum_{n}\left(\alpha_{n}^{k}+\sum_{\ell=1}^{k}(-1)^{\ell}\left(\begin{array}{c}
k-1 \\
\ell-1
\end{array}\right) \beta_{n}^{\ell}\right) \quad k \geq 2 .
\end{aligned}
$$

Remark 7.8. The set of $g$-positive specializations is larger than the set of Schur-positive specializations. Since the functions $g_{\lambda / \mu}$ are Schur-positive, every Schur-positive specialization is $g$ positive as well. However the converse is not true. For example, take the $g$-positive specialization $\rho=\psi_{\beta}: h_{n} \mapsto \beta \in(0,1)$ for all $n \geq 1$. Then $\rho\left(s_{\left(1^{2}\right)}\right)=\rho\left(e_{2}\right)=\rho\left(h_{1}^{2}-h_{2}\right)=\beta^{2}-\beta<0$.

\section{Two analogues of the Plancherel measure on partitions}

8.1. Corner growth model. Define a sequence of random partitions (or more concretely, their corresponding Young diagrams $)\left(\lambda^{(1)}, \lambda^{(2)}, \ldots\right)$ such that $\lambda^{(1)}=(1)$ and $\lambda^{(n+1)}$ is obtained from $\lambda^{(n)}$ by adding a box in one of its outer corners with equal probability, i.e. with probability $1 / \#\{$ outer corners $\}$. This Markov process is known as the corner growth model which can be viewed as a TASEP (see e.g. [Rom15]). Let

$$
p_{n}(\lambda):=\operatorname{Pr}\left(\lambda^{(n)}=\lambda\right) .
$$

As $n \rightarrow \infty$, the sequence $\left\{\lambda^{(n)}\right\}$ rescaled by $1 / \sqrt{n}$ has the following parabolic limit shape ${ }^{2}$

$$
\sqrt{x}+\sqrt{y}=6^{1 / 4} \text {. }
$$

${ }^{2}$ Formally, for any $\epsilon \in(0,1)$

$$
\operatorname{Pr}\left((1-\epsilon) \omega \subset\left[\lambda^{(n)}\right] \subset(1+\epsilon) \omega\right) \rightarrow 1 \text { as } n \rightarrow \infty,
$$

where $\omega=\left\{(x, y): x, y \geq 0, \sqrt{x}+\sqrt{y} \leq 6^{1 / 4}\right\}$ and $\left[\lambda^{(n)}\right]$ is the region under graph of partitions $\lambda^{(n)}$ rescaled by $1 / \sqrt{n}$ in both directions and drawn in French notation (upside down English). For more background on the corner growth model, its analysis, and connections with RSK, see [Rom15]. 
This result was first proved by Rost [Ros81]. The model was further analyzed by Johansson [Joh00] (cf. [Joh01]) making connections with RSK and longest increasing subsequences in generalized permutations.

Interestingly, this process is related to dual Grothendieck polynomials as follows. Let $\rho: \Lambda \rightarrow \mathbb{R}$ be a normalized $g$-positive specialization, i.e. $\rho\left(g_{(1)}\right)=1$. Then it is not difficult to show that

$$
\mu_{\rho, n}(\lambda):=p_{n}(\lambda) \rho\left(g_{\lambda}\right)
$$

is a probability measure on the set of partitions $\lambda \vdash n$.

In particular, take the specialization $\rho\left(g_{\lambda}\right)=g_{\lambda}(1)=1$, then as $n \rightarrow \infty$, partitions with respect to the measure $\mu_{\rho, n}(\lambda)=p_{n}(\lambda)$ have the above parabolic limit shape. Now we pose the same question for the Plancherel specialization $\rho=\pi: p_{1} \mapsto 1$ and $p_{k} \mapsto 0$ for $k \geq 2$. What is limit shape of partitions with respect to the measure $\mu_{\rho, n}(\lambda)=p_{n}(\lambda) \pi\left(g_{\lambda}\right)$ ?

Remark 8.1. The probabilities $p_{n}(\lambda)$ serve here as analogues of dimensions $f^{\lambda}$ for the classical Schur case with the Plancherel measure $f^{\lambda} \pi\left(s_{\lambda}\right)=\left(f^{\lambda}\right)^{2} / n$ !.

Remark 8.2. We address some further connections between the corner growth model and dual Grothendieck polynomials in [Yel19a].

8.2. Plancherel-Hecke measure. Let us introduce two more types of tableaux:

An increasing tableau is a filling of a Young diagram with positive integers strictly increasing both in rows and columns. Denote by $d^{\lambda}(n)$ the number of increasing tableaux of shape $\lambda$ filled with numbers from the set $\{1, \ldots, n\}$.

A standard set-valued tableau (SSVT) is a filling of a Young diagram with sets of positive integers so that entries strictly increase in both rows and columns. Denote by $e^{\lambda}(m)$ the number of SSVT filled with numbers $\{1, \ldots, m\}$.

Let $\varphi: \Gamma \rightarrow \mathbb{R}$ be a $G$-positive specialization of $\Gamma$. Then it is not difficult to show that

$$
M_{\varphi, n}(\lambda):=\frac{d^{\lambda}(n) \tilde{G}_{\lambda}(\varphi)}{\Delta^{n}}, \quad \Delta:=1+\tilde{G}_{(1)}(\varphi)
$$

is a probability measure on the set of partitions $\lambda \subset \delta_{n}:=(n, n-1, \ldots, 1)$.

In particular, take the Plancherel specialization $\varphi=\hat{\pi}_{\gamma}$. Then we can derive that

$$
\tilde{G}_{\lambda}\left(\hat{\pi}_{\gamma}\right)=\sum_{m} \frac{\gamma^{m}}{m !} e^{\lambda}(m)
$$

and the measure $M_{\varphi, n}(\lambda)$ specializes to the Plancherel-Hecke measure $\mu_{m, n}(\lambda)$ studied by Thomas and Yong in [TY11]. Namely, we have

$$
\mu_{m, n}(\lambda)=\frac{d^{\lambda}(n) e^{\lambda}(m)}{n^{m}}
$$

is a probability measure on the set of partitions $\lambda \subset \delta_{n}$ and $|\lambda| \leq m$. This result was obtained from a $K$-theoretic extension of RSK, the Hecke insertion algorithm [BKSTY08, TY09]. The measure is also naturally related to longest increasing subsequences of words of length $m$ in the alphabet $\{1, \ldots, n\}$. A special case of the conjecture made in [TY11] is that when $n=\Theta\left(m^{\alpha}\right)$ for $\alpha>1 / 2$, the partitions with respect to this measure have the same limit shape as for the Plancherel measure obtained by Vershik and Kerov [VK85], and Logan and Shepp [LS77]. 


\section{ACKNowledgements}

I am grateful to Askar Dzhumadil'daev, Igor Pak, Leonid Petrov, and Pavlo Pylyavskyy for many helpful conversations. I am also grateful to the referee for helpful remarks.

\section{REFERENCES}

[ASW52] M. Aissen, I. J. Schoenberg, and A. Whitney, On generating functions of totally positive sequences, I, J. d'Analyse Math. 2 (1952), 93-103.

[BO00] A. Borodin and G. Olshanski, Harmonic functions on multiplicative graphs and interpolation polynomials, Electronic J. Combin. 7.1 (2000): 28.

[BO17] A. Borodin and G. Olshanski, Representations of the infinite symmetric group, Cambridge Studies in Advanced Mathematics, Vol. 160, Cambridge University Press, 2017.

[Buc02] A. Buch, A Littlewood Richardson rule for the K-theory of Grassmannians, Acta Math. 189 (2002), 37-78.

[Buc05] A. Buch, Combinatorial K-theory, Topics in cohomological studies of algebraic varieties, Birkhäuser Basel, 2005, 87-103.

[BKSTY08] A. Buch, A. Kresch, M. Shimozono, H. Tamvakis, and A. Yong, Stable Grothendieck polynomials and $K$-theoretic factor sequences, Math. Ann. 340 (2008), 359-382.

[Edr52] A. Edrei, On the generating functions of totally positive sequences II, J. Anal. Math. 88 (1952), $104-109$.

[Fom94] S. Fomin, Duality of graded graphs, J. Algebraic Combin. 3 (1994) 357-404.

[FK94] S. Fomin and A. Kirillov, Grothendieck polynomials and the Yang-Baxter equation, Proc. 6th Intern. Conf. on Formal Power Series and Algebraic Combinatorics, DIMACS, (1994), 183-190.

[FZ00] S. Fomin and A. Zelevinsky, Total positivity: tests and parametrizations, Math. Intelligencer 22 (2000), 23-33.

[Ful97] W. Fulton, Young Tableaux, With applications to representation theory and geometry, Cambridge University Press, Cambridge, 1997.

[Gal17] P. Galashin, A Littlewood-Richardson rule for dual stable Grothendieck polynomials, J. Combin. Theory Ser. A 151 (2017), 23-35.

[GP92] M. Gasca and J. M. Peña, Total positivity and Neville elimination, Linear Algebra Appl. 165 (1992), 25-44.

[GV89] I. Gessel and X. Viennot, Determinants, paths, and plane partitions, preprint 1989.

[GO06] A. Gnedin and G. Olshanski, Coherent permutations with descent statistic and the boundary problem for the graph of zigzag diagrams, Int. Math. Res. Not. 2006 (2006).

[Joh00] K. Johansson, Shape fluctuations and random matrices, Commun. Math. Phys. 209 (2000), $437-476$.

[Joh01] K. Johansson, Random growth and random matrices, European Congress of Mathematics, Birkhäuser, Basel, 2001.

[KOO98] S. Kerov, A. Okounkov, and G. Olshanski, The boundary of the Young graph with Jack edge multiplicities, Int. Math. Res. Not. 4 (1998), 173-199.

[KV80] S. Kerov and A. Vershik, Characters, factor representations and K-functor of the infinite symmetric group. In: Operator Algebras and Group Representations, Vol. II (Neptun, 1980), Monographs and Studies in Mathematics, vol. 18, Pitman, Massachusetts, 1984, 23-32.

[Ker03] S.V. Kerov, Asymptotic representation theory of the symmetric group and its applications in analysis, Translations of Mathematical Monographs vol. 219, AMS, 2003.

[LP07] T. Lam and P. Pylyavskyy, Combinatorial Hopf algebras and K-homology of Grassmannians, Int. Math. Res. Not. Vol. 2007, (2007), rnm 125.

[Len00] C. Lenart, Combinatorial aspects of the K-theory of Grassmannians, Ann. Comb. 4 (2000), 67-82.

[LS83] A. Lascoux and M.-P. Schutzenberger, Symmetry and flag manifolds, Lecture Notes in Mathematics, Vol. 996 (1983), 118-144.

[LS77] B. Logan and L. Shepp, A variational problem for random Young tableaux, Adv. Math. 26 (1977), $206-222$. 
[Mac98] I. G. Macdonald, Symmetric functions and Hall-Littlewood polynomials, Oxford Univ. Press, Oxford (1998).

[Mat19] K. Matveev, Macdonald-positive specializations of the algebra of symmetric functions: Proof of the Kerov conjecture, Ann. Math. 189 (2019), 277-316.

[Naz90] M. Nazarov, Factor-representations of the infinite spin-symmetric group, Zap. Nauchn. Sem. LOMI, 1990, Vol. 181, 132-145.

[OR03] A. Okounkov and N. Reshetikhin, Correlation function of Schur process with application to local geometry of a random 3-dimensional Young diagram, J. Amer. Math. Soc. 16 (2003), 581-603.

[PP16] R. Patrias and P. Pylyavskyy, Combinatorics of K-theory via a K-theoretic Poirier-Reutenauer bialgebra, Discrete Math. 339 (2016), 1095-1115.

[PP18] R. Patrias and P. Pylyavskyy, Dual filtered graphs, Algebraic Combinatorics 1.4 (2018), 441-500.

[Rom15] D. Romik, The surprising mathematics of longest increasing subsequences, Cambridge Univ. Press, New York, 2015.

[Ros81] H. Rost, Nonequilibrium behaviour of a many particle process: density profile and local equilibria. Z. Wahrsch. Verw. Gebiete, 58 (1981), 41-53.

[Sch34] I. J. Schoenberg, Zur Abzählung der reellen Wurzeln algebraischer Gleichungen, Math. Z. 38 (1934), $546-564$.

[Sta88] R. Stanley, Differential posets, J. Amer. Math. Soc. 1 (1988), 919-961.

[Sta99] R. Stanley, Enumerative Combinatorics, Vol 2, Cambridge, (1999).

[Tho64] E. Thoma, Die unzerlegbaren, positiv-definiten Klassenfunktionen der abzahlbar unendlichen, symmetrischen Gruppe, Math. Z. 85 (1964), 40-61.

[TY09] H. Thomas and A. Yong, A jeu de taquin theory for increasing tableaux, with applications to $K$-theoretic Schubert calculus, Algebra \& Number Theory 3 (2009), 121-148.

[TY11] H. Thomas and A. Yong, Longest increasing subsequences, Plancherel-type measure and the Hecke insertion algorithm, Adv. Appl. Math. 46 (2011), 610-642.

[VK81] A. Vershik and S. Kerov, Asymptotic theory of characters of the symmetric group, Funct. Anal. Appl. 15 (1981), no. 4, 246-255.

[VK85] A. Vershik and S. Kerov, Asymptotic of the largest and the typical dimensions of irreducible representations of a symmetric group, Funct. Anal. Appl. 19 (1985), 21-31.

[Yel17] D. Yeliussizov, Duality and deformations of stable Grothendieck polynomials, J. Algebraic Combin. 45 (2017), 295-344.

[Yel19] D. Yeliussizov, Symmetric Grothendieck polynomials, skew Cauchy identities, and dual filtered Young graphs, J. Combin. Theory Ser. A 161 (2019), 453-485.

[Yel19a] D. Yeliussizov, Random plane partitions and corner distributions, arXiv:1910.13378, 2019.

[Yel19b] D. Yeliussizov, Enumeration of plane partitions by descents, arXiv:1911.03259, 2019.

KBTU, Almaty, Kazakhstan

E-mail address: yeldamir@gmail.com 